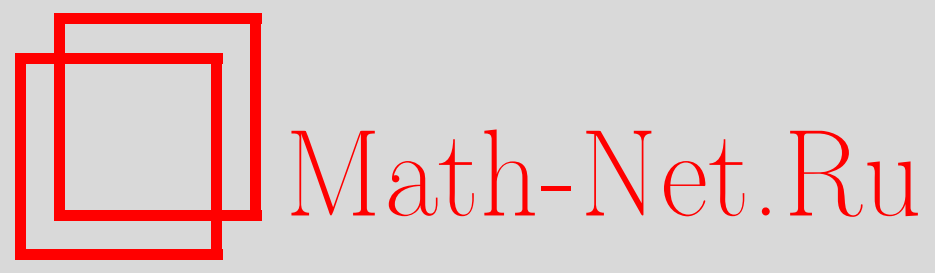

В. В. Кочергин, О сложности вычисления пары одночленов от двух переменных, Дискрет. матем., 2005, том 17, выпуск 4, 116-142

DOI: https://doi.org/10.4213/dm135

Использование Общероссийского математического портала Math-Net.Ru подразумевает, что вы прочитали и согласны с пользовательским соглашением http://www . mathnet.ru/rus/agreement

Параметры загрузки:

IP : 54.162 .127 .20

26 апреля 2023 г., 16:07:15

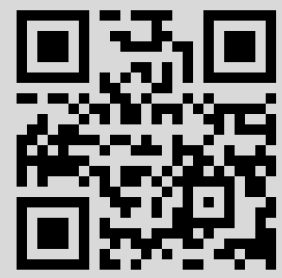




\title{
О сложности вычисления пары одночленов от двух переменных
}

\author{
() 2005 г. . В. Вочергин
}

Изучается обобщение задачи об эффективном вычислении степени $x^{n}$ по заданным $x$ и $n$ (или эквивалентной ей задачи о минимальной аддитивной цепочке для числа $n$ ), где $n \in \mathbf{N}$.

Сложность вычисления системы одночленов $\left\{x^{a} y^{b}, x^{c} y^{d}\right\}$, то есть минимальное число операций умножения, достаточное для вычисления (допускается многократное использование промежуточных результатов) по переменным $x$ и $y$, а также заданным показателям $a, b, c$ и $d$ системы одночленов $\left\{x^{a} y^{b}, x^{c} y^{d}\right\}$, обозначим через $l\left(x^{a} y^{b}, x^{c} y^{d}\right)$.

В работе доказано, что при выполнении условия $\max \{a, b, c, d\} \rightarrow \infty$, справедливо соотношение

$$
l\left(x^{a} y^{b}, x^{c} y^{d}\right) \sim \log _{2}(|a d-b c|+a+b+c+d) .
$$

Работа выполнена при поддержке Российского фонда фундаментальный исследований, проект 05-01-00994, программой Президента Российской Федерации поддержки ведущих научных школ, проект НШ-1807.2003.1, и программой Университеты России, проект УР.04.02.528.

\section{1. Введение}

Рассматривается обобщение задачи о сложности возведения в степень, то есть задачи о нахождении минимального числа $l\left(x^{n}\right)$ операций умножения, достаточного для вычисления по переменной $x$ величины $x^{n}$. Эту задачу (а также ее обобщения) часто рассматривают в аддитивной постановке, это известная задача об аддитивных цепочках, которая формулируется следующим образом (см., например, [1]). Аддитивной цепочкой для натурального числа $n$ называется любая последовательность целых чисел $a_{0}=1, a_{1}, \ldots, a_{r}=n$, обладающая следующим свойством: для каждого $k, 1 \leqslant k \leqslant r$, найдутся два целых числа (не обязательно различных) $i$ и $j, 0 \leqslant i \leqslant k-1,0 \leqslant j \leqslant k-1$, таких, что $a_{k}=a_{i}+a_{j}$. Длиной такой аддитивной цепочки называется число $r$. Очевидно, что минимальная длина адцитивной цепочки для $n$ равна $l\left(x^{n}\right)$.

Для величины $l\left(x^{n}\right)$ А. Брауэром [2] была установлена асимптотическая формула

$$
l\left(x^{n}\right) \sim \log n
$$

(здесь и далее $\log x$ означает $\log _{2} x$ ), а также была получена верхняя оценка

$$
l\left(x^{n}\right) \leqslant \log n+\frac{\log n}{\log \log n}+O\left(\frac{\log n \log \log \log n}{(\log \log n)^{2}}\right) .
$$


Р. Беллман в [3] сформулировал задачу о сложности вычисления монома (одночлена) от $m$ переменных, то есть нахождения величины $l\left(x_{1}^{n_{1}} x_{2}^{n_{2}} \ldots x_{m}^{n_{m}}\right)$. На языке аддитивных цепочек эта задача формулируется как задача о нахождении минимальной длины адयитивной цепочки векторов (наборов) для вектора (набора) $\left(n_{1}, n_{2}, \ldots, n_{m}\right)$, где аддитивная цепочка векторов для вектора $\left(n_{1}, n_{2}, \ldots, n_{m}\right)$ - это последовательность векторов, начинающаяся с $m$ единичных векторов $(1,0, \ldots, 0),(0,1, \ldots, 0), \ldots,(0,0, \ldots, 1)$ и заканчивающаяся вектором $\left(n_{1}, n_{2}, \ldots, n_{m}\right)$, причем каждый элемент последовательности является (покомпонентной) суммой двух каких-либо предшествующих.

Д. Кнут (см. [1], разд.4.6.3., упр.32) поставил задачу о сложности вычисления $m$ степеней одной переменной, то есть нахождения величины $l\left(x^{n_{1}}, x^{n_{2}}, \ldots, x^{n_{m}}\right)$. На языке аддитивных цепочек эта задача формулируется как задача о нахождении минимальной длины аддитивной цепочки, содержащей в себе все элементы набора $\left(n_{1}, n_{2}, \ldots, n_{m}\right)$.

В [4] показано, что для любого фиксированного $m$

$$
l\left(x_{1}^{n_{1}} x_{2}^{n_{2}} \ldots x_{m}^{n_{m}}\right) \sim \log \left(\max \left\{n_{1}, \ldots, n_{m}\right\}\right)
$$

при $\max \left\{n_{1}, \ldots, n_{m}\right\} \rightarrow \infty$.

В [5] установлено, что для любого фиксированного $m$

$$
l\left(x^{n_{1}}, x^{n_{2}}, \ldots, x^{n_{m}}\right) \sim \log \left(\max \left\{n_{1}, \ldots, n_{m}\right\}\right)
$$

при $\max \left\{n_{1}, \ldots, n_{m}\right\} \rightarrow \infty$.

Позже в работах $[6,7,8]$ при слабых ограничениях было получено асимптотически точное решение задач Беллмана и Кнута.

Дальнейшим обобщением задачи об эффективном возведении в степень (а также обобщением задач Беллмана и Кнута) является задача о нахождении сложности вычисления системы мономов от многих переменных, то есть задача нахождения величины $l\left(x_{1}^{n_{11}} x_{2}^{n_{12}} \ldots x_{q}^{n_{1 q}}, x_{1}^{n_{21}} x_{2}^{n_{22}} \ldots x_{q}^{n_{2 q}}, \ldots, x_{1}^{n_{p 1}} x_{2}^{n_{p 2}} \ldots x_{q}^{n_{p q}}\right)$.

На языке аддитивных цепочек эта задача формулируется как задача о нахождении минимальной длины $r$ аддитивной цепочки $q$-мерных векторов (наборов) вида $(1,0, \ldots, 0)$, $(0,1, \ldots, 0), \ldots,(0,0, \ldots, 1), a_{1}, a_{2}, \ldots, a_{r}$, начинающейся с $q$ единичных векторов и удовлетворяющей следующим условиям:

(1) для каждого $k, 1 \leqslant k \leqslant r$, найдутся два целых числа (не обязательно различных) $i$ и $j, 0 \leqslant i \leqslant k-1,0 \leqslant j \leqslant k-1$, таких, что $a_{k}=a_{i}+a_{j}$ (сложение векторов покомпонентное);

(2) $\left\{\left(n_{11}, n_{12}, \ldots, n_{1 q}\right),\left(n_{21}, n_{22}, \ldots, n_{2 q}\right), \ldots,\left(n_{p 1}, n_{p 2}, \ldots, n_{p q}\right)\right\} \subseteq\left\{a_{1}, a_{2}, \ldots, a_{r}\right\}$.

Очевидно, что минимальная длина $r$ такой аддитивной цепочки равна

$$
l\left(x_{1}^{n_{11}} x_{2}^{n_{12}} \ldots x_{q}^{n_{1 q}}, x_{1}^{n_{21}} x_{2}^{n_{22}} \ldots x_{q}^{n_{2 q}}, \ldots, x_{1}^{n_{p 1}} x_{2}^{n_{p 2}} \ldots x_{q}^{n_{p q}}\right)
$$

В общем виде задача о нахождении асимптотики роста сложности вычисления системы мономов (с ростом, например, суммы всех показателей степеней) представляется очень трудной.

Стоит сказать, что асимптотический рост величины $L(p, q, k)$, максимума сложности вычисления системы мономов, где максимум берется по всем системам из $p$ мономов от $q$ переменных с показателями степеней не превосходящими $k$, при слабых ограничениях был установлен в [9]. 
Отдельные оценки сложности вычислений систем мономов можно найти в статьяx [10]-[13].

Для дальнейшего продвижения в изучении этой задачи представляется важным исследовать асимптотическое поведение сложности вычисления систем мономов малой размерности, то есть небольшого фиксированного числа мономов от нескольких переменных.

В данной работе исследуется сложность вычисления системы из двух мономов от двух переменных. Для этого случая найдена асимптотика роста сложности. Основным результатом является следующее утверждение.

Теорема 1. Пусть

$$
a_{n}=\max \left\{a_{n}, b_{n}, c_{n}, d_{n}\right\}, \quad n=1,2, \ldots,
$$

$u a_{n} \rightarrow \infty$ nрu $n \rightarrow \infty$. Тогдa

$$
l\left(x^{a_{n}} y^{b_{n}}, x^{c_{n}} y^{d_{n}}\right) \sim \log \left(\left|a_{n} d_{n}-b_{n} c_{n}\right|+a_{n}\right) .
$$

\section{2. Нижняя оценка}

При доказательстве нижней оценки удобно в качестве модели вычислений использовать схемы из функциональных элементов (см., например, [14] и [15]), имеющие два входа, на которые подаются переменные $x$ и $y$, соответственно, а все элементы схемы являются двухвходовыми и реализуют произведение мономов, подаваемых на входы. Сложность $l(S)$ схемы $S$ - это число функциональных элементов в этой схеме. Очевидно, что

$$
l\left(x^{a} y^{b}, x^{c} y^{d}\right)=\min l(S),
$$

где минимум берется по всем схемам, реализующим систему мономов $\left\{x^{a} y^{b}, x^{c} y^{d}\right\}$ (здесь и далее считаем, что $a+b>0$ и $c+d>0$ ).

Кроме этого, определим обобщенную схему как схему из функщиональных элементов с двумя входами, на которые подаются переменные $x$ и $y$, соответственно, а элементы схемы подразделяются на двухвходовые и одновходовые; двухвходовые элементы реализуют произведение подаваемых на их входы мономов, а каждый одновходовый элемент реализует для некоторого $r$ (вообще говоря, своего для каждого элемента), $r \in \mathbf{Q}, 1<r \leqslant 2$, $r$-ю степень подаваемого на вход элемента монома. Строго говоря, при определении обобщенной схемы следует говорить обобщенный моном, а не моном, так как показатели степеней у переменных уже, вообще говоря, не натуральные, а рациональные, однако здесь и далее во избежание громоздкости терминологии будем использовать слово моном.

Сложность $\lambda(S)$ обобщенной схемы $S$ - это число функщиональных элементов в $S$. Положим

$$
\lambda\left(x^{a} y^{b}, x^{c} y^{d}\right)=\min \lambda(S),
$$

где минимум берется по всем обобщенным схемам, реализующим систему мономов $\left\{x^{a} y^{b}, x^{c} y^{d}\right\}$. Нетрудно понять, что минимум действительно достигается (как альтернативный вариант можно в обобщенной схеме для системы мономов $\left\{x^{a} y^{b}, x^{c} y^{d}\right\}$ разрешить использовать среди одновходовых элементов только такие элементы возведения в степень, у которых на показатель степени $r$, кроме условий $r \in \mathbf{Q}, 1<r \leqslant 2$, наложено дополнительное ограничение: в представлении $r$ в виде несократимой дроби $p / q$ число $q$ не превосходит величины $\left.\max \left\{a_{n}, b_{n}, c_{n}, d_{n}\right\}\right)$. 
Очевидно, что

$$
l\left(x^{a} y^{b}, x^{c} y^{d}\right) \geqslant \lambda\left(x^{a} y^{b}, x^{c} y^{d}\right),
$$

поэтому достаточно доказать нужную нижнюю оценку для величины $\lambda\left(x^{a} y^{b}, x^{c} y^{d}\right)$.

Сначала найдем величину $\lambda\left(x^{a}\right)$ (формально следовало бы говорить о величине $\left.\lambda\left(x^{a} y^{0}, x^{0} y^{1}\right)\right)$.

Лемма 1. Пусть $a \in \mathbf{Q}, a \geqslant 1$. Тогд $a$

$$
\lambda\left(x^{a}\right)=\lceil\log a\rceil .
$$

Доказательство. То, что $\lambda\left(x^{a}\right) \leqslant\lceil\log a\rceil$, очевидно. Нижняя оценка, совпадающая с верхней, непосредственно вытекает из того факта, что с помошью $k$ элементов можно реализовать только степень с показателем, не превышающим $2^{k}$.

Теперь сведем задачу о нижней оценке величины $\lambda\left(x^{a} y^{b}, x^{c} y^{d}\right)$ в общем случае к случаю, когда показатели степени переменных одного из мономов равны.

Лемма 2. Пусть $a, b, c, d$ - рачиональные числа, $a \geqslant b \geqslant 1, c \in\{0\} \cup[1, \infty)$, $d \in\{0\} \cup[1, \infty)$. Тогда

$$
\lambda\left(x^{a} y^{b}, x^{c} y^{d}\right) \geqslant \lambda\left(x^{a} y^{a}, x^{c} y^{d a / b}\right)-\lceil\log (a / b)\rceil .
$$

Доказательство. Рассмотрим минимальную обобщенную схему $S_{1}$, вычисляющую систему $\left\{x^{a} y^{b}, x^{c} y^{d}\right\}, a \geqslant b \geqslant 1$. На один из входов схемы $S_{1}$ вместо переменной $y$ подадим выход некоторой минимальной схемы $S_{2}$, вычисляющей моном $y^{a / b}$. После такого преобразования на выходах получившейся схемы будут реализованы мономы $x^{a}\left(y^{a / b}\right)^{b}=x^{a} y^{a}$ и $x^{c}\left(y^{a / b}\right)^{d}=x^{c} y^{d a / b}$. Поэтому

$$
\lambda\left(x^{a} y^{a}, x^{c} y^{d a / b}\right) \leqslant \lambda\left(S_{1}\right)+\lambda\left(S_{2}\right)=\lambda\left(y^{a / b}\right)+\lambda\left(x^{a} y^{b}, x^{c} y^{d}\right) .
$$

Используя лемму 1 , получаем, что

$$
\lambda\left(x^{a} y^{b}, x^{c} y^{d}\right) \geqslant \lambda\left(x^{a} y^{a}, x^{c} y^{d a / b}\right)-\lceil\log (a / b)\rceil .
$$

Лемма 2 доказана.

Пусть $v$ - некоторая вершина обобщенной схемы (соответствующая либо входу схемы, либо операции умножения, либо операции возведения в степень $r, 1<r \leqslant 2$ ). Обозначим через $\partial(z, v)$, где $z \in\{x, y\}$, степень переменной $z$ в мономе, реализуемом (вычисляемом) в вершине $v$.

Каждой обобщенной схеме $S$ с двумя входами $x$ и $y$ сопоставим число $k_{1}(S)$ следующим образом:

$$
k_{1}(S)=\max _{v \in S}|\partial(x, v)-\partial(y, v)| .
$$

Далее каждой паре вершин $\left(v_{1}, v_{2}\right), v_{1} \in S, v_{2} \in S$, припишем число $k_{2}\left(v_{1}, v_{2}\right)$ следующим образом.

(1) Если выполняется неравенство

$$
\left(\partial\left(x, v_{1}\right)-\partial\left(y, v_{1}\right)\right)\left(\partial\left(x, v_{2}\right)-\partial\left(y, v_{2}\right)\right)<0,
$$


то полагаем

$$
\begin{aligned}
k_{2}\left(v_{1}, v_{2}\right)=\frac{\max \left(\partial\left(x, v_{1}\right), \partial\left(y, v_{1}\right)\right) \max \left(\partial\left(x, v_{2}\right), \partial\left(y, v_{2}\right)\right)}{\max \left(\left|\partial\left(x, v_{1}\right)-\partial\left(y, v_{1}\right)\right|,\left|\partial\left(x, v_{2}\right)-\partial\left(y, v_{2}\right)\right|\right)} \\
\\
\quad-\frac{\min \left(\partial\left(x, v_{1}\right), \partial\left(y, v_{1}\right)\right) \min \left(\partial\left(x, v_{2}\right), \partial\left(y, v_{2}\right)\right)}{\max \left(\left|\partial\left(x, v_{1}\right)-\partial\left(y, v_{1}\right)\right|,\left|\partial\left(x, v_{2}\right)-\partial\left(y, v_{2}\right)\right|\right)}
\end{aligned}
$$

(2) Если выполняются равенства

$$
\partial\left(x, v_{1}\right)-\partial\left(y, v_{1}\right)=0, \quad \partial\left(x, v_{2}\right)-\partial\left(y, v_{2}\right)=0
$$

то полагаем

$$
k_{2}\left(v_{1}, v_{2}\right)=\partial\left(x, v_{1}\right)+\partial\left(x, v_{2}\right)
$$

(3) Во всех остальных случаях полагаем

$$
k_{2}\left(v_{1}, v_{2}\right)=0
$$

Рассмотрим случай, когда выполняется неравенство

$$
\left(\partial\left(x, v_{1}\right)-\partial\left(y, v_{1}\right)\right)\left(\partial\left(x, v_{2}\right)-\partial\left(y, v_{2}\right)\right)<0 .
$$

Без ограничения общности будем считать, что $\partial\left(x, v_{1}\right)>\partial\left(y, v_{1}\right)$. Тогда, с одной стороны,

$$
\begin{aligned}
k_{2}\left(v_{1}, v_{2}\right)= & \frac{\partial\left(x, v_{1}\right) \partial\left(y, v_{2}\right)-\partial\left(x, v_{1}\right) \partial\left(x, v_{2}\right)+\partial\left(x, v_{1}\right) \partial\left(x, v_{2}\right)-\partial\left(y, v_{1}\right) \partial\left(x, v_{2}\right)}{\max \left(\left|\partial\left(x, v_{1}\right)-\partial\left(y, v_{1}\right)\right|,\left|\partial\left(x, v_{2}\right)-\partial\left(y, v_{2}\right)\right|\right)} \\
= & \partial\left(x, v_{1}\right) \frac{\partial\left(y, v_{2}\right)-\partial\left(x, v_{2}\right)}{\max \left(\left|\partial\left(x, v_{1}\right)-\partial\left(y, v_{1}\right)\right|,\left|\partial\left(x, v_{2}\right)-\partial\left(y, v_{2}\right)\right|\right)} \\
& \quad+\partial\left(x, v_{2}\right) \frac{\partial\left(x, v_{1}\right)-\partial\left(y, v_{1}\right)}{\max \left(\left|\partial\left(x, v_{1}\right)-\partial\left(y, v_{1}\right)\right|,\left|\partial\left(x, v_{2}\right)-\partial\left(y, v_{2}\right)\right|\right)},
\end{aligned}
$$

а с другой стороны,

$$
\begin{aligned}
k_{2}\left(v_{1}, v_{2}\right)= & \frac{\partial\left(x, v_{1}\right) \partial\left(y, v_{2}\right)-\partial\left(y, v_{1}\right) \partial\left(y, v_{2}\right)+\partial\left(y, v_{1}\right) \partial\left(y, v_{2}\right)-\partial\left(y, v_{1}\right) \partial\left(x, v_{2}\right)}{\max \left(\left|\partial\left(x, v_{1}\right)-\partial\left(y, v_{1}\right)\right|,\left|\partial\left(x, v_{2}\right)-\partial\left(y, v_{2}\right)\right|\right)} \\
= & \partial\left(y, v_{1}\right) \frac{\partial\left(y, v_{2}\right)-\partial\left(x, v_{2}\right)}{\max \left(\left|\partial\left(x, v_{1}\right)-\partial\left(y, v_{1}\right)\right|,\left|\partial\left(x, v_{2}\right)-\partial\left(y, v_{2}\right)\right|\right)} \\
& +\partial\left(y, v_{2}\right) \frac{\partial\left(x, v_{1}\right)-\partial\left(y, v_{1}\right)}{\max \left(\left|\partial\left(x, v_{1}\right)-\partial\left(y, v_{1}\right)\right|,\left|\partial\left(x, v_{2}\right)-\partial\left(y, v_{2}\right)\right|\right)}
\end{aligned}
$$

Отметим, что коэффициент, соответствующий вершине с меньшим модулем разности показателей степеней переменных, равен единище, а коэффициент, соответствующий вершине с большим модулем разности показателей степеней переменных, лежит в интервале $(0,1]$, то есть либо справедливы равенства

$$
k_{2}\left(v_{1}, v_{2}\right)=\partial\left(x, v_{1}\right)+\alpha \partial\left(x, v_{2}\right)=\partial\left(y, v_{1}\right)+\alpha \partial\left(y, v_{2}\right)
$$

либо равенства

$$
k_{2}\left(v_{1}, v_{2}\right)=\alpha \partial\left(x, v_{1}\right)+\partial\left(x, v_{2}\right)=\alpha \partial\left(y, v_{1}\right)+\partial\left(y, v_{2}\right) \text {, }
$$


где

$$
\alpha=\frac{\min \left(\left|\partial\left(x, v_{1}\right)-\partial\left(y, v_{1}\right)\right|,\left|\partial\left(x, v_{2}\right)-\partial\left(y, v_{2}\right)\right|\right)}{\max \left(\left|\partial\left(x, v_{1}\right)-\partial\left(y, v_{1}\right)\right|,\left|\partial\left(x, v_{2}\right)-\partial\left(y, v_{2}\right)\right|\right)},
$$

причем $\alpha$ удовлетворяет неравенствам $0<\alpha \leqslant 1$.

Таким образом, в случае, когда выполняется неравенство

$$
\left(\partial\left(x, v_{1}\right)-\partial\left(y, v_{1}\right)\right)\left(\partial\left(x, v_{2}\right)-\partial\left(y, v_{2}\right)\right)<0
$$

величина $k_{2}\left(v_{1}, v_{2}\right)$ численно равна показателю степеней переменных монома от двух переменных с одинаковыми показателями степеней, который можно получить из мономов, вычисляемых в вершинах $v_{1}$ и $v_{2}$, путем умножения одного из них на некоторую степень $\alpha, \alpha \in(0,1]$, другого монома.

Очевидно, что в случае, когда выполняются равенства $\partial\left(x, v_{1}\right)-\partial\left(y, v_{1}\right)=0$ и $\partial\left(x, v_{2}\right)-\partial\left(y, v_{2}\right)=0$, величина $k_{2}\left(v_{1}, v_{2}\right)$ численно равна максимально возможному показателю степеней переменных монома от двух переменных с одинаковыми показателями степеней, который можно получить из мономов, вычисляемых в вершинах $v_{1}$ и $v_{2}$, путем умножения одного из них на некоторую степень $\alpha, \alpha \in(0,1]$, другого монома.

Теперь сопоставим произвольной обобщенной схеме $S$ с двумя входами еще один параметр, параметр $k_{2}(S)$, следующим образом:

$$
k_{2}(S)=\max k_{2}\left(v_{1}, v_{2}\right),
$$

где максимум берется по всем парам вершин (вершины могут и совпадать) схемы $S$.

Отметим, что в обобщенной схеме, все элементы которой вычисляют попарно различные мономы, если выполняется равенство $k_{2}\left(v_{1}, v_{2}\right)=k_{2}(S)$, то либо $v_{1}=v_{2}$, либо $\left(\partial\left(x, v_{1}\right)-\partial\left(y, v_{1}\right)\right)\left(\partial\left(x, v_{2}\right)-\partial\left(y, v_{2}\right)\right)<0$.

Заметим также, что в случае, когда $v_{1}$ - вход схемы, соответствуюший переменной $x$, а $v_{2}$ - вход схемы, соответствуюший переменной $y$, выполняется неравенство $k_{2}\left(v_{1}, v_{2}\right) \geqslant 1$. Поэтому для любой обобщенной схемы $S$ справедливо соотношение $k_{2}(S) \geqslant 1$.

Учитывая вышесказанное, получаем, что

$$
\begin{aligned}
k_{2}(S)=\max \left\{p \mid \exists\left(v_{1}, v_{2}, \alpha\right): v_{1} \in S, v_{2} \in S, \alpha \in(0,1]\right. & \\
& \left.p=\partial\left(x, v_{1}\right)+\alpha \partial\left(x, v_{2}\right)=\partial\left(y, v_{1}\right)+\alpha \partial\left(y, v_{2}\right)\right\}
\end{aligned}
$$

Таким образом, $k_{2}(S)$ дает, в некотором смысле, верхнюю оценку потенциала обобщенной схемы $S$ по отношению к получению монома с равными показателями степеней как можно большей степени. Некоторая громоздкость при определении параметра $k_{2}(S)$ связана с тем, что для дальнейшего нужна равномерность роста параметра $k_{2}$ при добавления к схеме новых элементов, то есть чтобы при добавлении небольшого числа элементов не происходил резкий рост величины $k_{2}$.

В следующей лемме исследуемая величина $\lambda(S)$ оценивается снизу через произведение введенных параметров $k_{1}(S)$ и $k_{2}(S)$. Положим

$$
K(S)=k_{1}(S) k_{2}(S) .
$$

Лемма 3. Пусть $S$ - обобщенная схема с двумя входами. Тогда

$$
\lambda(S) \geqslant \log K(S) .
$$


Доказательство. Индукщией по $\lambda(S)$ установим справедливость соотношения

$$
K(S) \leqslant 2^{\lambda(S)} .
$$

Если $\lambda(S)=0$, то есть в схеме $S$ нет элементов умножения или возведения в степень, то $k_{1}(S)=1, k_{2}(S)=1$, и утверждение леммы выполняется.

Рассмотрим произвольную обобщенную схему $S$, на входы которой подаются переменные $x$ и $y$. Обозначим через $v_{0}$ некоторую висячую вершину (то есть вершину, в которой вычисляется моном, который уже не подается на вход никакого элемента схемы), например, один из выходов схемы $S$. Через $S^{\prime}$ обозначим обобщенную схему, получающуюся из схемы $S$ путем исключения из нее вершины $v_{0}$ и ребер, инщидентных вершине $v_{0}$. Покажем, что $K(S) \leqslant 2 K\left(S^{\prime}\right)$.

Пусть в вершине $v_{0}$ вычисляется моном $x^{a_{0}} y^{b_{0}}$. Без ограничения общности можно считать, что $a_{0} \geqslant b_{0}$.

Если справедливо равенство $k_{2}(S)=k_{2}\left(S^{\prime}\right)$, то, учитывая очевидное неравенство $k_{1}(S) \leqslant 2 k_{1}\left(S^{\prime}\right)$, получаем нужное соотношение $K(S) \leqslant 2 K\left(S^{\prime}\right)$. Поэтому далее считаем, что $k_{2}(S)>k_{2}\left(S^{\prime}\right)$.

Обозначим через $v_{1}$ вершину (вершина $v_{1}$ может, вообще говоря, совпадать с вершиной $v_{0}$ ), на которой достигает максимума при фиксированной вершине $v_{0}$ величина $k_{2}\left(v_{0}, v\right)$, а вычисляемый в вершине $v_{1}$ моном обозначим через $x^{a_{1}} y^{b_{1}}$. В силу того, что $k_{2}(S)>k_{2}\left(S^{\prime}\right)$, справедливо равенство $k_{2}\left(v_{0}, v_{1}\right)=k_{2}(S)$.

Теперь отдельно рассмотрим два случая, в зависимости от того, какая операция приписана вершине $v_{0}$.

Случай 1. Вершине $v_{0}$ приписана операция возведения в степень $r, r \in(1,2]$. Обозначим моном, который в степени $r$ дает моном $x^{a_{0}} y^{b_{0}}$, через $x^{a_{2}} y^{b_{2}}$, а вершину, в которой этот моном вычисляется через $v_{2}$. Очевидно, что

$$
a_{0}=r a_{2}, \quad b_{0}=r b_{2} .
$$

Случай 1.1. Пусть выполняется равенство $a_{0}=b_{0}$. Тогда

$$
k_{1}(S)=k_{1}\left(S^{\prime}\right), \quad k_{2}(S)=k_{2}\left(v_{0}, v_{0}\right) \leqslant r k_{2}\left(S^{\prime}\right)
$$

Поэтому

$$
K(S) \leqslant 2 K\left(S^{\prime}\right)
$$

Случай 1.2. Пусть выполняется неравенство $a_{0}>b_{0}$. Тогда показатели степеней переменных вычисляемого в вершине $v_{1}$ монома $x^{a_{1}} y^{b_{1}}$ удовлетворяют соотношению

$$
a_{1}<b_{1} \text {. }
$$

Случай 1.2.1. Пусть выполняется неравенство $b_{1}-a_{1} \geqslant b_{0}-a_{0}$. Тогда, во-первых,

$$
k_{1}(S)=k_{1}\left(S^{\prime}\right)
$$

а во-вторых, учитывая неравенства

$$
b_{1}-a_{1} \geqslant a_{0}-b_{0}=r\left(a_{2}-b_{2}\right)>a_{2}-b_{2},
$$


получаем, что

$$
\begin{aligned}
k_{2}(S) & =k_{2}\left(v_{0}, v_{1}\right)=\frac{a_{0} b_{1}-b_{0} a_{1}}{b_{1}-a_{1}}=\frac{r a_{2} b_{1}-r b_{2} a_{1}}{b_{1}-a_{1}} \\
& =r k_{2}\left(v_{1}, v_{2}\right) \leqslant r k_{2}\left(S^{\prime}\right) .
\end{aligned}
$$

Поэтому

$$
K(S) \leqslant r K\left(S^{\prime}\right) \leqslant 2 K\left(S^{\prime}\right)
$$

Случай 1.2.2. Пусть выполняется неравенство $b_{1}-a_{1}<a_{0}-b_{0}$. Тогда

$$
k_{2}(S)=k_{2}\left(v_{0}, v_{1}\right)=\frac{a_{0} b_{1}-b_{0} a_{1}}{a_{0}-b_{0}}=\frac{r a_{2} b_{1}-r b_{2} a_{1}}{r\left(a_{2}-b_{2}\right)}=\frac{a_{2} b_{1}-b_{2} a_{1}}{a_{2}-b_{2}}
$$

Случай 1.2.2.1. Пусть выполняется неравенство $b_{1}-a_{1} \leqslant a_{2}-b_{2}$. Тогда

$$
k_{2}(S)=\frac{a_{2} b_{1}-b_{2} a_{1}}{a_{2}-b_{2}}=k_{2}\left(v_{1}, v_{2}\right) \leqslant k_{2}\left(S^{\prime}\right) \text {. }
$$

Следовательно,

$$
K(S) \leqslant 2 K\left(S^{\prime}\right)
$$

Случай 1.2.2.2. Пусть выполняется неравенство $b_{1}-a_{1}>a_{2}-b_{2}$. Тогда

$$
\begin{aligned}
k_{2}(S) & =\frac{a_{2} b_{1}-b_{2} a_{1}}{a_{2}-b_{2}}=\frac{b_{1}-a_{1}}{a_{2}-b_{2}} \frac{a_{2} b_{1}-b_{2} a_{1}}{b_{1}-a_{1}} \\
& =\frac{b_{1}-a_{1}}{a_{2}-b_{2}} k_{2}\left(v_{1}, v_{2}\right) \leqslant \frac{b_{1}-a_{1}}{a_{2}-b_{2}} k_{2}\left(S^{\prime}\right) .
\end{aligned}
$$

Поэтому

$$
\begin{aligned}
K(S) & =k_{1}(S) k_{2}(S) \leqslant \frac{a_{0}-b_{0}}{b_{1}-a_{1}} k_{1}\left(S^{\prime}\right) \frac{b_{1}-a_{1}}{a_{2}-b_{2}} k_{2}\left(S^{\prime}\right) \\
& =\frac{a_{0}-b_{0}}{a_{2}-b_{2}} k_{1}\left(S^{\prime}\right) k_{2}\left(S^{\prime}\right)=r k_{1}\left(S^{\prime}\right) k_{2}\left(S^{\prime}\right) \leqslant 2 K\left(S^{\prime}\right) .
\end{aligned}
$$

Таким образом, случай 1 разобран полностью.

Случай 2. Вершине $v_{0}$ приписана операция умножения. Обозначим мономы, произведение которых вычисляется в вершине $v_{0}$, через $x^{a_{2}} y^{b_{2}}$ и $x^{a_{3}} y^{b_{3}}$, а вершины, в которых они вычисляется, через $v_{2}$ и $v_{3}$ соответственно. Очевидно, что

$$
a_{0}=a_{2}+a_{3}, \quad b_{0}=b_{2}+b_{3} .
$$

Без ограничения общности будем считать, что

$$
a_{2}-b_{2} \geqslant a_{3}-b_{3} \text {. }
$$


Случай 2.1. Пусть выполняется равенство $a_{0}=b_{0}$. Тогда

$$
k_{1}(S)=k_{1}\left(S^{\prime}\right)
$$

и вследствие неравенства $k_{2}(S)>k_{2}\left(S^{\prime}\right)$, справедливы равенства

$$
k_{2}(S)=k_{2}\left(v_{0}, v_{0}\right)=2 a_{0} .
$$

Поэтому

$$
\begin{aligned}
K(S) & =k_{1}(S) k_{2}(S)=k_{1}\left(S^{\prime}\right) 2 a_{0}=k_{1}\left(S^{\prime}\right) 2 k_{2}\left(v_{2}, v_{3}\right) \\
& \leqslant 2 k_{1}\left(S^{\prime}\right) k_{2}\left(S^{\prime}\right)=2 K(S)
\end{aligned}
$$

Случай 2.2. Пусть выполняется неравенство $a_{0}>b_{0}$. Тогда показатели степеней переменных вычисляемого в вершине $v_{1}$ монома $x^{a_{1}} y^{b_{1}}$ удовлетворяют соотношению

$$
a_{1}<b_{1} \text {. }
$$

Случай 2.2.1. Пусть выполняется неравенство $a_{0}-b_{0} \leqslant b_{1}-a_{1}$. Тогда, во-первых,

$$
k_{1}(S)=k_{1}\left(S^{\prime}\right)
$$

а во-вторых,

$$
\begin{aligned}
k_{2}(S) & =k_{2}\left(v_{0}, v_{1}\right)=\frac{a_{0} b_{1}-b_{0} a_{1}}{b_{1}-a_{1}} \\
& =\frac{\left(a_{2}+a_{3}\right) b_{1}-\left(b_{2}+b_{3}\right) a_{1}}{b_{1}-a_{1}}=\frac{a_{2} b_{1}-a_{1} b_{2}}{b_{1}-a_{1}}+\frac{a_{3} b_{1}-a_{1} b_{3}}{b_{1}-a_{1}} .
\end{aligned}
$$

Случай 2.2.1.1. Пусть выполняется неравенство $a_{3}-b_{3} \geqslant 0$. Тогда $a_{2}-b_{2}>0$, поскольку $a_{2}-b_{2} \geqslant a_{3}-b_{3}$ и $a_{0}-b_{0}>0$. Далее,

$$
b_{1}-a_{1} \geqslant a_{0}-b_{0} \geqslant a_{2}-b_{2} \geqslant a_{3}-b_{3} \geqslant 0 \text {. }
$$

Поэтому

$$
\frac{a_{2} b_{1}-a_{1} b_{2}}{b_{1}-a_{1}}=k_{2}\left(v_{1}, v_{2}\right) \leqslant k_{2}\left(S^{\prime}\right) \text {. }
$$

Если $a_{3}-b_{3}=0$, то

$$
\frac{a_{3} b_{1}-a_{1} b_{3}}{b_{1}-a_{1}}=a_{3}<k_{2}\left(v_{3}, v_{3}\right) \leqslant k_{2}\left(S^{\prime}\right) .
$$

Если $a_{3}-b_{3}>0$, то

$$
\frac{a_{3} b_{1}-a_{1} b_{3}}{b_{1}-a_{1}}=k_{2}\left(v_{1}, v_{3}\right) \leqslant k_{2}\left(S^{\prime}\right) \text {. }
$$

Таким образом,

$$
k_{2}(S) \leqslant \frac{a_{2} b_{1}-a_{1} b_{2}}{b_{1}-a_{1}}+\frac{a_{3} b_{1}-a_{1} b_{3}}{b_{1}-a_{1}} \leqslant 2 k_{2}\left(S^{\prime}\right),
$$

и, в силу равенства $k_{1}(S)=k_{1}\left(S^{\prime}\right)$, получаем, что

$$
K(S) \leqslant K\left(S^{\prime}\right) \text {. }
$$


Случай 2.2.1.2. Пусть выполняется неравенство $a_{3}-b_{3}<0$. Представим величину $k_{2}(S)$ в виде

$$
k_{2}(S)=k_{2}\left(v_{0}, v_{1}\right)=a_{0}+\frac{a_{0}-b_{0}}{b_{1}-a_{1}} a_{1}
$$

Оценим эту величину сверху через сумму значений $k_{2}\left(v_{1}, v_{2}\right)$ и $k_{2}\left(v_{2}, v_{3}\right)$.

Из неравенства $a_{0}>b_{0}$ следует неравенство $a_{2}-b_{2}>b_{3}-a_{3}$. Поэтому в условиях случая 2.2.1.2

$$
k_{2}\left(v_{2}, v_{3}\right)=a_{2} \frac{b_{3}-a_{3}}{a_{2}-b_{2}}+a_{3} .
$$

Далее, чтобы найти $k_{2}\left(v_{1}, v_{2}\right)$ рассмотрим два случая.

Случай 2.2.1.2.1. Пусть выполняется неравенство $b_{1}-a_{1} \leqslant a_{2}-b_{2}$. Тогда

$$
k_{2}\left(v_{1}, v_{2}\right)=a_{1}+\frac{b_{1}-a_{1}}{a_{2}-b_{2}}
$$

В силу неравенства $b_{1}-a_{1} \geqslant a_{0}-b_{0}$ получаем, что

$$
b_{1}-a_{1} \geqslant\left(a_{2}-b_{2}\right)+\left(a_{3}-b_{3}\right) \text {, }
$$

и следовательно,

$$
a_{2}-b_{2} \leqslant\left(b_{1}-a_{1}\right)+\left(b_{3}-a_{3}\right) .
$$

Учитывая это неравенство и неравенство $a_{0}-b_{0} \leqslant b_{1}-a_{1}$, получаем, что

$$
\begin{aligned}
k_{2}(S)=k_{2}\left(v_{0}, v_{1}\right) & =a_{0}+\frac{a_{0}-b_{0}}{b_{1}-a_{1}} a_{1} \leqslant a_{1}+a_{2}+a_{3} \\
& \leqslant a_{1}+a_{2} \frac{\left(b_{1}-a_{1}\right)+\left(b_{3}-a_{3}\right)}{a_{2}-b_{2}}+a_{3} \\
& =\left(a_{1}+a_{2} \frac{b_{1}-a_{1}}{a_{2}-b_{2}}\right)+\left(a_{2} \frac{b_{3}-a_{3}}{a_{2}-b_{2}}+a_{3}\right) \\
& =k_{2}\left(v_{1}, v_{2}\right)+k_{2}\left(v_{2}, v_{3}\right) \leqslant 2 k_{2}\left(S^{\prime}\right) .
\end{aligned}
$$

Таким образом, учитывая равенство $k_{1}(S)=k_{1}\left(S^{\prime}\right)$, получаем, что

$$
K(S) \leqslant 2 K\left(S^{\prime}\right)
$$

Случай 2.2.1.2.2. Пусть выполняется неравенство $b_{1}-a_{1}>a_{2}-b_{2}$. Тогда

$$
k_{2}\left(v_{1}, v_{2}\right)=a_{1} \frac{a_{2}-b_{2}}{b_{1}-a_{1}}+a_{2}
$$

С использованием неравенств

$$
b_{3}-a_{3} \geqslant 0, \quad a_{0}-b_{0} \leqslant a_{2}-b_{2}
$$


(последнее неравенство следует из того же соотношения $b_{3}-a_{3} \geqslant 0$ ) получаем, что

$$
\begin{aligned}
k_{2}(S)=k_{2}\left(v_{0}, v_{1}\right) & =a_{0}+a_{1} \frac{a_{0}-b_{0}}{b_{1}-a_{1}}=a_{1} \frac{a_{0}-b_{0}}{b_{1}-a_{1}}+a_{2}+a_{3} \\
& \leqslant a_{1} \frac{a_{2}-b_{2}}{b_{1}-a_{1}}+a_{2}\left(1+\frac{b_{3}-a_{3}}{a_{2}-b_{2}}\right)+a_{3} \\
& =\left(a_{1} \frac{a_{2}-b_{2}}{b_{1}-a_{1}}+a_{2}\right)+\left(a_{2} \frac{b_{3}-a_{3}}{a_{2}-b_{2}}+a_{3}\right) \\
& =k_{2}\left(v_{1}, v_{2}\right)+k_{2}\left(v_{2}, v_{3}\right) \leqslant 2 k_{2}\left(S^{\prime}\right) .
\end{aligned}
$$

Таким образом, учитывая равенство $k_{1}(S)=k_{1}\left(S^{\prime}\right)$, получаем, что

$$
K(S) \leqslant 2 K\left(S^{\prime}\right)
$$

Случай 2.2.2. Пусть выполняется неравенство $a_{0}-b_{0}>b_{1}-a_{1}$. В этом случае справедливы равенства

$$
k_{2}(S)=k_{2}\left(v_{0}, v_{1}\right)=\frac{a_{0} b_{1}-a_{1} b_{0}}{a_{0}-b_{0}}=a_{0} \frac{b_{1}-a_{1}}{a_{0}-b_{0}}+a_{1}
$$

Случай 2.2.2.1. Пусть выполняется неравенство $a_{3}-b_{3} \geqslant 0$. Тогда вместе с неравенством $b_{1}-a_{1} \leqslant a_{0}-b_{0}$ справедливы соотношения

$$
a_{2}-b_{2} \leqslant a_{0}-b_{0}, \quad a_{3}-b_{3}<a_{0}-b_{0},
$$

вытекающие из соотношений

$$
a_{2}-b_{2} \geqslant a_{3}-b_{3}, \quad\left(a_{2}-b_{2}\right)+\left(a_{3}-b_{3}\right)=a_{0}-b_{0} .
$$

Поэтому

$$
k_{1}(S) \leqslant \frac{a_{0}-b_{0}}{\max \left(b_{1}-a_{1}, a_{2}-b_{2}, a_{3}-b_{3}\right)} k_{1}\left(S^{\prime}\right)
$$

Тогда

$$
\begin{aligned}
K(S)=k_{1}(S) k_{2}(S) & \leqslant \frac{a_{0}-b_{0}}{\max \left(b_{1}-a_{1}, a_{2}-b_{2}, a_{3}-b_{3}\right)} k_{1}\left(S^{\prime}\right) \frac{a_{0} b_{1}-a_{1} b_{0}}{a_{0}-b_{0}} \\
& =\frac{\left(a_{2} b_{1}-a_{1} b_{2}\right)+\left(a_{3} b_{1}-a_{1} b_{3}\right)}{\max \left(b_{1}-a_{1}, a_{2}-b_{2}, a_{3}-b_{3}\right)} k_{1}\left(S^{\prime}\right) \\
& \leqslant\left(\frac{a_{2} b_{1}-a_{1} b_{2}}{\max \left(b_{1}-a_{1}, a_{2}-b_{2}\right)}+\frac{a_{3} b_{1}-a_{1} b_{3}}{\max \left(b_{1}-a_{1}, a_{3}-b_{3}\right)}\right) k_{1}\left(S^{\prime}\right) .
\end{aligned}
$$

В силу соотношений

$$
a_{0}>b_{0}, \quad a_{2}-b_{2} \geqslant a_{3}-b_{3} \geqslant 0, \quad a_{0}-b_{0}=\left(a_{2}-b_{2}\right)+\left(a_{3}-b_{3}\right),
$$

справедливо неравенство $a_{2}>b_{2}$. Поэтому

$$
\frac{a_{2} b_{1}-a_{1} b_{2}}{\max \left(b_{1}-a_{1}, a_{2}-b_{2}\right)}=k_{2}\left(v_{1}, v_{2}\right) \leqslant k_{2}\left(S^{\prime}\right) .
$$


Покажем, что справедливо неравенство

$$
\frac{a_{3} b_{1}-a_{1} b_{3}}{\max \left(b_{1}-a_{1}, a_{3}-b_{3}\right)} \leqslant k_{2}\left(S^{\prime}\right) \text {. }
$$

Действительно, если $a_{3}=b_{3}$, то

$$
\frac{a_{3} b_{1}-a_{1} b_{3}}{\max \left(b_{1}-a_{1}, a_{3}-b_{3}\right)}=\frac{a_{3}\left(b_{1}-a_{1}\right)}{b_{1}-a_{1}}=a_{3}=k_{2}\left(v_{3}, v_{3}\right) / 2<k_{2}\left(S^{\prime}\right) .
$$

Если же выполняется неравенство $a_{3}-b_{3}>0$, то

$$
\frac{a_{3} b_{1}-a_{1} b_{3}}{\max \left(b_{1}-a_{1}, a_{3}-b_{3}\right)}=k_{2}\left(v_{1}, v_{3}\right) \leqslant k_{2}\left(S^{\prime}\right) \text {. }
$$

Возвращаясь к оценке величины $K(S)$, получаем, что

$$
\begin{aligned}
K(S) & \leqslant\left(\frac{a_{2} b_{1}-a_{1} b_{2}}{\max \left(b_{1}-a_{1}, a_{2}-b_{2}\right)}+\frac{a_{3} b_{1}-a_{1} b_{3}}{\max \left(b_{1}-a_{1}, a_{3}-b_{3}\right)}\right) k_{1}\left(S^{\prime}\right) \\
& \leqslant 2 k_{2}\left(S^{\prime}\right) k_{1}\left(S^{\prime}\right)=2 K\left(S^{\prime}\right) .
\end{aligned}
$$

Случай 2.2.2.2. Пусть выполняется неравенство $a_{3}-b_{3}<0$. Тогда

$$
a_{2}-b_{2} \geqslant a_{0}-b_{0} \text {, }
$$

и следовательно,

$$
k_{1}(S)=k_{1}\left(S^{\prime}\right)
$$

Отметим, что выполняются неравенства

$$
a_{2}-b_{2}>b_{1}-a_{1}, \quad a_{2}-b_{2}>b_{3}-a_{3}
$$

так как $\left.a_{0}>b_{0}\right)$. Поэтому

$$
k_{2}\left(v_{1}, v_{2}\right)=a_{1}+a_{2} \frac{b_{1}-a_{1}}{a_{2}-b_{2}}, \quad k_{2}\left(v_{2}, v_{3}\right)=a_{2} \frac{b_{3}-a_{3}}{a_{2}-b_{2}}+a_{3} .
$$

Далее, используя неравенства

$$
b_{1}-a_{1}<a_{0}-b_{0}, \quad b_{3}-a_{3}>0,
$$

получаем, что

$$
\frac{b_{1}-a_{1}}{a_{0}-b_{0}} \leqslant \frac{\left(b_{1}-a_{1}\right)+\left(b_{3}-a_{3}\right)}{\left(a_{0}-b_{0}\right)+\left(b_{3}-a_{3}\right)}=\frac{b_{1}-a_{1}}{a_{2}-b_{2}}+\frac{b_{3}-a_{3}}{a_{2}-b_{2}} .
$$

Тогда

$$
\begin{aligned}
k_{2}(S) & =k_{2}\left(v_{0}, v_{1}\right)=a_{0} \frac{b_{1}-a_{1}}{a_{0}-b_{0}}+a_{1}=a_{1}+\left(a_{2}+a_{3}\right) \frac{b_{1}-a_{1}}{a_{0}-b_{0}} \\
& \leqslant a_{1}+a_{2} \frac{b_{1}-a_{1}}{a_{0}-b_{0}}+a_{3} \leqslant a_{1}+a_{2}\left(\frac{b_{1}-a_{1}}{a_{2}-b_{2}}+\frac{b_{3}-a_{3}}{a_{2}-b_{2}}\right)+a_{3} \\
& =\left(a_{1}+a_{2} \frac{b_{1}-a_{1}}{a_{2}-b_{2}}\right)+\left(a_{2} \frac{b_{3}-a_{3}}{a_{2}-b_{2}}+a_{3}\right) \\
& =k_{2}\left(v_{1}, v_{2}\right)+k_{2}\left(v_{2}, v_{3}\right) \leqslant 2 k_{2}\left(S^{\prime}\right) .
\end{aligned}
$$


Поэтому

$$
K(S) \leqslant 2 K\left(S^{\prime}\right)
$$

Утверждение леммы 3 доказано во всех случаях.

Теперь с помощью доказанной леммы оценим снизу сложность вычисления системы из двух мономов от двух переменных обобщенными схемами в случае, когда показатели степени переменных одного из мономов равны.

Лемма 4. Пусть $a, c, d$-рачиональные числа, $a \geqslant 1, c \in\{0\} \cup[1, \infty), d \in\{0\} \cup[1, \infty)$. Тогда

$$
\lambda\left(x^{a} y^{a}, x^{c} y^{d}\right) \geqslant \log a+\log (\max (|c-d|, 1))+1 .
$$

Доказательство. Пусть обобщенная схема $S$, которая вычисляет систему мономов $\left\{x^{a} y^{a}, x^{c} y^{d}\right\}$, удовлетворяет условию $\lambda(S)=\lambda\left(x^{a} y^{a}, x^{c} y^{d}\right)$. Тогда для величин $k_{1}(S)$ и $k_{2}(S)$ справедливы оценки

$$
k_{1}(S) \geqslant \max (|c-d|, 1), \quad k_{2}(S) \geqslant 2 a .
$$

Теперь, применяя лемму 3 , получаем, что

$$
\begin{aligned}
\lambda\left(x^{a} y^{a}, x^{c} y^{d}\right) & =\lambda(S) \geqslant \log K(S)=\log \left(k_{1}(S) k_{2}(S)\right) \\
& \geqslant \log (2 a \max (|c-d|, 1))=\log a+\log (\max |c-d|, 1)+1 .
\end{aligned}
$$

Лемма 4 доказана.

Перейдем теперь непосредственно к доказательству нужной нижней оценки.

Лемма 5. Пусть $a, b, c, d-$ целые неотрицательные числа, $a=\max \{a, b, c, d\}$. Тогда

$$
\lambda\left(x^{a} y^{b}, x^{c} y^{d}\right) \geqslant \log (|a d-b c|+a)-1 \text {. }
$$

Доказательство. Отдельно рассмотрим несколько случаев.

Случай 1. Пусть выполняется условие $b=0$. В этом случае будем считать, что $d>0$, иначе система мономов не будет содержать степеней переменной $y$ (но утверждение леммы при этом все равно в силу леммы 1 будет справедливо).

Случай 1.1. Пусть выполняется условие $c=0$. Тогда, применяя лемму 1 , получаем, что

$$
\lambda\left(x^{a} y^{b}, x^{c} y^{d}\right)=\lambda\left(x^{a}, y^{d}\right) \geqslant\lceil\log a\rceil+\lceil\log d\rceil \geqslant \log (a d)=\log |a d-b c| .
$$

Случай 1.2. Пусть выполняется условие $c>0$.

Случай 1.2.1. Пусть выполняется условие $c \geqslant d$. Тогда, применяя леммы 2 и 4 , получаем, что

$$
\begin{aligned}
\lambda\left(x^{a} y^{b}, x^{c} y^{d}\right) & =\lambda\left(x^{c} y^{d}, x^{a}\right) \geqslant \lambda\left(x^{c} y^{c}, x^{a}\right)-\lceil\log (c / d)\rceil \\
& \geqslant \log c+\log (\max (a, 1))+1-\lceil\log (c / d)\rceil \\
& \geqslant \log (a d)=\log |a d-b c|
\end{aligned}
$$


Случай 1.2.2. Пусть выполняется условие $c<d$. Аналогично, применяя леммы 2 и 4 , получаем, что

$$
\begin{aligned}
\lambda\left(x^{a} y^{b}, x^{c} y^{d}\right) & =\lambda\left(x^{c} y^{d}, x^{a}\right) \geqslant \lambda\left(x^{d} y^{d}, x^{a d / c}\right)-\lceil\log (d / c)\rceil \\
& \geqslant \log d+\log (\max (a d / c, 1))+1-\lceil\log (d / c)\rceil \\
& \geqslant \log (a d)=\log |a d-b c| .
\end{aligned}
$$

Случай 2. Пусть выполняется условие $b>0$. Опять, применяя леммы 2 и 4, получаем, что

$$
\begin{aligned}
\lambda\left(x^{a} y^{b}, x^{c} y^{d}\right) & \geqslant \lambda\left(x^{a} y^{a}, x^{c} y^{d a / b}\right)-\lceil\log (a / b)\rceil \\
& \geqslant \log a+\log (\max |c-d a / b|, 1)+1-\lceil\log (a / b)\rceil \\
& \geqslant \log (\max (|a d-b c|, b)) .
\end{aligned}
$$

Таким образом, во всех случаях показано, что

$$
\lambda\left(x^{a} y^{b}, x^{c} y^{d}\right) \geqslant \log (\max (|a d-b c|, 1)) .
$$

С другой стороны, справедливо очевидное неравенство

$$
\lambda\left(x^{a} y^{b}, x^{c} y^{d}\right) \geqslant \log a .
$$

Объединяя обе оценки, получаем, что

$$
\begin{aligned}
\lambda\left(x^{a} y^{b}, x^{c} y^{d}\right) & \geqslant \log (\max (|a d-b c|, a)) \\
& \geqslant \log \left(\frac{1}{2}(|a d-b c|+a)\right)=\log (|a d-b c|+a)-1
\end{aligned}
$$

Лемма 5 доказана.

Нижняя оценка теоремы в силу неравенства

$$
l\left(x^{a} y^{b}, x^{c} y^{d}\right) \geqslant \lambda\left(x^{a} y^{b}, x^{c} y^{d}\right)
$$

следует непосредственно из леммы 5.

\section{3. Верхняя оценка}

Предложим конкретный способ вычисления мономов $x^{a} y^{b}, x^{c} y^{d}$, где $a=\max \{a, b, c, d\}$.

В основе совместного вычисления мономов $x^{a} y^{b}$ и $x^{c} y^{d}$ лежит обобщение конструкция из [2] (см. также [1]) для вычисления одной степени $x^{a}$.

Пусть $u$ - натуральный параметр, значение которого определим позже.

Представим числа $a$ и $c$ в системе счисления по основанию $2^{u}$. Пусть значность полученных представлений равна, соответственно, $s$ и $t$. Тогда

$$
\begin{array}{lll}
a=\alpha_{0}+\alpha_{1} 2^{u}+\ldots+\alpha_{s-1} 2^{(s-1) u}, & 0 \leqslant \alpha_{i}<2^{u}, \quad i=0,1, \ldots, s-1, \quad \alpha_{s-1} \geqslant 1, \\
c=\gamma_{0}+\gamma_{1} 2^{u}+\ldots+\gamma_{t-1} 2^{(t-1) u}, & 0 \leqslant \gamma_{i}<2^{u}, \quad i=0,1, \ldots, t-1, \quad \gamma_{t-1} \geqslant 1 .
\end{array}
$$


Отметим, что при этом справедливы соотношения

$$
(s-1) u \leqslant \log a<s u, \quad(t-1) u \leqslant \log c<t u .
$$

Далее положим

$$
p_{i}=\left\lfloor\frac{b}{a} 2^{i u}\right\rfloor, \quad i=0,1, \ldots, s-1
$$

Покажем, что

$$
2^{u} p_{i} \leqslant p_{i+1} \leqslant 2^{u} p_{i}+2^{u}-1, \quad i=0,1, \ldots, s-2
$$

Действительно, с одной стороны,

$$
\frac{p_{i+1}}{p_{i}}=\frac{\left\lfloor(b / a) 2^{(i+1) u}\right\rfloor}{\left\lfloor(b / a) 2^{i u}\right\rfloor} \geqslant \frac{\left.\left\lfloor(b / a) 2^{i u} t\right\rfloor 2^{u}\right\rfloor}{\left\lfloor(b / a) 2^{i u}\right\rfloor}=2^{u},
$$

а с другой,

$$
p_{i+1}-2^{u} p_{i}=\left\lfloor\frac{b}{a} 2^{i u} 2^{u}\right\rfloor-\left\lfloor\frac{b}{a} 2^{i u}\right\rfloor 2^{u}<\frac{b}{a} 2^{i u} 2^{u}-\left(\frac{b}{a} 2^{i u}-1\right) 2^{u}=2^{u},
$$

и следовательно, учитывая целочисленность величины $p_{i+1}-2^{u} p_{i}$, находим, что $p_{i+1}-2^{u} p_{i} \leqslant 2^{u}-1$.

Рассмотрим отдельно два случая.

В первом случае, пусть выполнено неравенство $a d \geqslant b c$. Положим $d^{\prime}=\lfloor b c / a\rfloor$. Заметим, что тогда верно соотношение $d^{\prime} \leqslant d$.

Положим

$$
\hat{b}=\alpha_{0} p_{0}+\alpha_{1} p_{1}+\ldots+\alpha_{s-1} p_{s-1}
$$

Тогда, во-первых,

$$
\begin{aligned}
\hat{b} & =\alpha_{0}\left\lfloor\frac{b}{a}\right\rfloor+\alpha_{1}\left\lfloor\frac{b}{a} 2^{u}\right\rfloor+\ldots+\alpha_{s-1}\left\lfloor\frac{b}{a} 2^{(s-1) u}\right\rfloor \\
& \leqslant \frac{b}{a}\left(\alpha_{0}+\alpha_{1} 2^{u}+\ldots+\alpha_{s-1} 2^{(s-1) u}\right)=b,
\end{aligned}
$$

а во-вторых,

$$
\begin{aligned}
\hat{b} & =\alpha_{0}\left\lfloor\frac{b}{a}\right\rfloor+\alpha_{1}\left\lfloor\frac{b}{a} 2^{u}\right\rfloor+\ldots+\alpha_{s-1}\left\lfloor\frac{b}{a} 2^{(s-1) u}\right\rfloor \\
& \geqslant \alpha_{0}\left(\frac{b}{a}-1\right)+\alpha_{1}\left(\frac{b}{a} 2^{u}-1\right)+\ldots+\alpha_{s-1}\left(\frac{b}{a} 2^{(s-1) u}-1\right) \\
& =b-\left(\alpha_{0}+\alpha_{1}+\ldots+\alpha_{s-1}\right)>b-2^{u} s .
\end{aligned}
$$

Таким образом,

$$
b-s 2^{u}<\hat{b} \leqslant b
$$

Положим

$$
\hat{d}^{\prime}=\gamma_{0} p_{0}+\gamma_{1} p_{1}+\ldots+\gamma_{t-1} p_{t-1} .
$$


Для величины $\hat{d}^{\prime}$ докажем аналогичные неравенства. С одной стороны,

$$
\begin{aligned}
\hat{d}^{\prime}= & \gamma_{0}\left\lfloor\frac{b}{a}\right\rfloor+\gamma_{1}\left\lfloor\frac{b}{a} 2^{u}\right\rfloor+\ldots+\gamma_{t-1}\left\lfloor\frac{b}{a} 2^{(t-1) u}\right\rfloor \\
& \leqslant \frac{b}{a}\left(\gamma_{0}+\gamma_{1} 2^{u}+\ldots+\gamma_{t-1} 2^{(t-1) u}\right)=\frac{b}{a} c,
\end{aligned}
$$

и учитывая целочисленность величины $\hat{d}^{\prime}$, получаем неравенство

$$
\hat{d}^{\prime} \leqslant\lfloor b c / a\rfloor=d^{\prime}
$$

С другой стороны,

$$
\begin{aligned}
\hat{d}^{\prime} & =\gamma_{0}\left\lfloor\frac{b}{a}\right\rfloor+\gamma_{1}\left\lfloor\frac{b}{a} 2^{u}\right\rfloor+\ldots+\gamma_{t-1}\left\lfloor\frac{b}{a} 2^{(t-1) u}\right\rfloor \\
& \geqslant \gamma_{0}\left(\frac{b}{a}-1\right)+\gamma_{1}\left(\frac{b}{a} 2^{u}-1\right)+\ldots+\gamma_{t-1}\left(\frac{b}{a} 2^{(t-1) u}-1\right) \\
& =\frac{b}{a} c-\left(\gamma_{0}+\gamma_{1}+\ldots+\gamma_{t-1}\right)>\left\lfloor\frac{b}{a} c\right\rfloor-t 2^{u}=d^{\prime}-t 2^{u} .
\end{aligned}
$$

Следовательно,

$$
d^{\prime}-t 2^{u}<\hat{d}^{\prime} \leqslant d^{\prime}
$$

Перейдем непосредственно к описанию процесса совместного вычисления мономов $x^{a} y^{b}$ и $x^{c} y^{d}$ в условиях рассматриваемого случая. Это вычисление будет состоять из пяти этапов. Обозначим через $l_{1 i}, i=1,2,3,4,5$, число операций умножения, используемых на $i$-м этапе.

I этап. Вычисление системы степеней

$$
y, y^{2}, y^{3}, \ldots, y^{2^{u}-1}
$$

Очевидно, что

$$
l_{11}=2^{u}-2
$$

II этап. Вычисление мономов

$$
x y^{p_{0}}, x^{2^{u}} y^{p_{1}}, \ldots, x^{2^{(s-1) u}} y^{p_{s-1}} .
$$

Сначала, учитывая, что $p_{0}$ равно либо 0 , либо 1 , последовательно вычисляем

$$
x y^{p_{0}},\left(x y^{p_{0}}\right)^{2},\left(x y^{p_{0}}\right)^{4}, \ldots,\left(x y^{p_{0}}\right)^{2^{u}} .
$$

Теперь, учитывая справедливость неравенств $p_{0} 2^{u} \leqslant p_{1} \leqslant p_{r} 2^{u}+2^{u}-1$, а также тот факт, что все степени $y$, не превышающие величины $2^{u}-1$, уже вычислены на первом этапе, с использованием одной операции умножения можем получить моном

$$
x^{2^{u}} y^{p_{1}}=x^{2^{u}} y^{p_{0} 2^{u}} y^{p_{1}-p_{0} 2^{u}}=\left(x y^{p_{0}}\right)^{2^{u}} y^{p_{1}-p_{0} 2^{u}}
$$


Далее аналогично последовательно вычисляем мономы

$$
\begin{gathered}
\left(x^{2^{u}} y^{p_{1}}\right)^{2},\left(x^{2^{u}} y^{p_{1}}\right)^{4}, \ldots,\left(x^{2^{u}} y^{p_{1}}\right)^{2^{u}}, x^{2^{2 u}} y^{p_{2}}, \\
\left(x^{2^{2 u}} y^{p_{2}}\right)^{2},\left(x^{2^{2 u}} y^{p_{2}}\right)^{4}, \ldots,\left(x^{2^{2 u}} y^{p_{2}}\right)^{2^{u}}, x^{2^{3 u}} y^{p_{3}}, \\
\ldots \\
\left(x^{2^{(s-2) u}} y^{p_{s-2}}\right)^{2},\left(x^{2^{(s-2) u}} y^{p_{s-2}}\right)^{4}, \ldots,\left(x^{2^{(s-2) u}} y^{p_{s-2}}\right)^{2^{u}}, x^{2^{(s-2) u}} y^{p_{s-1}} .
\end{gathered}
$$

Для каждого нового монома, получаемого на втором этапе, использовалась одна операция умножения. Поэтому $l_{12} \leqslant(s-1)(u+1)$. Поскольку $(s-1) u \leqslant \log a$, получаем, что

$$
l_{12} \leqslant s+\log a .
$$

III этап. Вычисление мономов

$$
x^{a} y^{\hat{b}}, \quad x^{c} y^{\hat{d}^{\prime}}
$$

Введем множества индексов $I_{1}, I_{2}, \ldots, I_{2^{u}-1}$ и $J_{1}, J_{2}, \ldots, J_{2^{u}-1}$ следующим образом:

$$
\begin{array}{ll}
I_{m}=\left\{i \mid(0 \leqslant i \leqslant s-1) \&\left(\alpha_{i}=m\right)\right\}, & m=1,2, \ldots, 2^{u}-1, \\
J_{m}=\left\{i \mid(0 \leqslant i \leqslant t-1) \&\left(\gamma_{i}=m\right)\right\}, & m=1,2, \ldots, 2^{u}-1 .
\end{array}
$$

Отметим, что хотя бы одно из множеств индексов $I_{m}, m=1,2, \ldots, 2^{u}-1$, непусто, так как $\alpha_{s-1} \neq 0$. Аналогично, непусто хотя бы одно из множеств $J_{m}, m=1,2, \ldots, 2^{u}-1$. Кроме того, отметим справедливость неравенств

$$
\sum_{i=1}^{2^{u}-1}\left|I_{i}\right| \leqslant s, \quad \sum_{i=1}^{2^{u}-1}\left|J_{i}\right| \leqslant t .
$$

Последовательно определим мономы

$$
f_{2^{u-1}}(x, y), g_{2^{u-1}}(x, y), f_{2^{u-2}}(x, y), g_{2^{u-2}}(x, y), \ldots, f_{1}(x, y), g_{1}(x, y) .
$$

Сначала положим

$$
\begin{aligned}
& f_{2^{u}-1}(x, y)=\prod_{i \in I_{2^{u}-1}}\left(x^{2^{i u}} y^{p_{i}}\right), \\
& g_{2^{u}-1}(x, y)=\prod_{i \in J_{2} u_{-1}}\left(x^{2^{i u}} y^{p_{i}}\right) .
\end{aligned}
$$

Далее, для $m=2^{u}-2,2^{u}-3, \ldots, 1$, положим

$$
\begin{aligned}
& f_{m}(x, y)=f_{m+1}(x, y) \prod_{i \in I_{m}}\left(x^{2^{i u}} y^{p_{i}}\right), \\
& g_{m}(x, y)=g_{m+1}(x, y) \prod_{i \in J_{m}}\left(x^{2^{i u}} y^{p_{i}}\right) .
\end{aligned}
$$


Теперь, считая, что произведение пустого множества сомножителей по определению равно единице, вычислим последовательно все отличные от единицы мономы

$$
\begin{aligned}
& \prod_{i \in I_{2} u-1}\left(x^{2^{i u}} y^{p_{i}}\right), \prod_{i \in I_{2} u_{-2}}\left(x^{2^{i u}} y^{p_{i}}\right), \ldots, \prod_{i \in I_{1}}\left(x^{2^{i u}} y^{p_{i}}\right), \\
& \prod_{i \in J_{2} u_{-1}}\left(x^{2^{i u}} y^{p_{i}}\right), \prod_{i \in J_{2} u_{-2}}\left(x^{2^{i u}} y^{p_{i}}, \ldots, \prod_{i \in J_{1}}\left(x^{2^{i u}} y^{p_{i}}\right) .\right.
\end{aligned}
$$

На вычисление этих мономов потребуется не более $s+t$ операций умножения.

Далее, используя по одной операции на каждый новый моном, вычислим

$$
\begin{gathered}
f_{2^{u}-1}(x, y), f_{2^{u}-2}(x, y), \ldots, f_{1}(x, y) ; \\
f_{2^{u}-1}(x, y) f_{2^{u}-2}(x, y), \\
f_{2^{u}-1}(x, y) f_{2^{u-2}}(x, y) f_{2^{u}-3}(x, y), \ldots, f_{2^{u}-1}(x, y) f_{2^{u}-2}(x, y) \ldots f_{1}(x, y) ; \\
g_{2^{u}-1}(x, y), g_{2^{u}-2}(x, y), \ldots, g_{1}(x, y) ; \\
g_{2^{u}-1}(x, y) g_{2^{u}-2}(x, y), \\
g_{2^{u}-1}(x, y) g_{2^{u}-2}(x, y) g_{2^{u}-3}(x, y), \ldots, g_{2^{u}-1}(x, y) g_{2^{u}-2}(x, y) \ldots g_{1}(x, y) .
\end{gathered}
$$

Тем самым вычислены мономы $x^{a} y^{\hat{b}}$ и $x^{c} y^{\hat{d}^{\prime}}$. Действительно,

$$
\begin{aligned}
& f_{2^{u-1}}(x, y) f_{2^{u-2}}(x, y) \ldots f_{1}(x, y) \\
& =\left(\prod_{i \in I_{2} u_{-1}}\left(x^{2^{i u}} y^{p_{i}}\right)\right)^{2^{u}-1}\left(\prod_{i \in I_{2} u_{-2}}\left(x^{2^{i u}} y^{p_{i}}\right)\right)^{2^{u}-2} \ldots\left(\prod_{i \in I_{1}}\left(x^{2^{i u}} y^{p_{i}}\right)\right)^{1} \\
& =\left(\prod_{i \in I_{2} u-1}\left(x^{2^{i u}} y^{p_{i}}\right)^{\alpha_{i}}\right)\left(\prod_{i \in I_{2} u_{-2}}\left(x^{2^{i u}} y^{p_{i}}\right)^{\alpha_{i}}\right) \ldots\left(\prod_{i \in I_{1}}\left(x^{2^{i u}} y^{p_{i}}\right)^{\alpha_{i}}\right) \\
& =\prod_{i: \alpha_{i} \neq 0}\left(x^{\alpha_{i} i^{i u}} y^{\alpha_{i} p_{i}}\right) \\
& =x^{\alpha_{0}+\alpha_{1} 2^{u}+\ldots+\alpha_{s-1} 2^{(s-1) u}} y^{\alpha_{0} p_{0}+\alpha_{1} p_{1}+\ldots+\alpha_{s-1} p_{s-1}}=x^{a} y^{\hat{b}} \text {, } \\
& g_{2^{u-1}}(x, y) g_{2^{u-2}}(x, y) \ldots g_{1}(x, y) \\
& =\left(\prod_{i \in J_{2} u_{-1}}\left(x^{2^{i u}} y^{p_{i}}\right)\right)^{2^{u}-1}\left(\prod_{i \in J_{2} u_{-2}}\left(x^{2^{i u}} y^{p_{i}}\right)\right)^{2^{u}-2} \cdots\left(\prod_{i \in J_{1}}\left(x^{2^{i u}} y^{p_{i}}\right)\right)^{1} \\
& =\left(\prod_{i \in J_{2} u_{-1}}\left(x^{2^{i u}} y^{p_{i}}\right)^{\gamma_{i}}\right)\left(\prod_{i \in J_{2} u-2}\left(x^{2^{i u}} y^{p_{i}}\right)^{\gamma_{i}}\right) \ldots\left(\prod_{i \in J_{1}}\left(x^{2^{i u}} y^{p_{i}}\right)^{\gamma_{i}}\right) \\
& =\prod_{i: \gamma_{i} \neq 0}\left(x^{\gamma_{i} 2^{i u}} y^{\gamma_{i} p_{i}}\right) \\
& =x^{\gamma_{0}+\gamma_{1} 2^{u}+\ldots+\gamma_{t-1} 2^{(t-1) u}} y^{\gamma_{0} p_{0}+\gamma_{1} p_{1}+\ldots+\gamma_{t-1} p_{t-1}}=x^{c} y^{\hat{d}^{\prime}} .
\end{aligned}
$$

Очевидно, что

$$
l_{13} \leqslant\left(s+2 \cdot 2^{u}\right)+\left(t+2 \cdot 2^{u}\right) \leqslant 2 s+4 \cdot 2^{u} .
$$


IV этап. Вычисление степеней

$$
y^{d^{\prime}-\hat{d}^{\prime}}, \quad y^{b-\hat{b}}, \quad y^{d-d^{\prime}}
$$

Учитывая соотношения

$$
0 \leqslant d^{\prime}-\hat{d}^{\prime} \leqslant t 2^{u} \leqslant s 2^{u}, \quad 0 \leqslant b-\hat{b} \leqslant s 2^{u},
$$

а также используя очевидное неравенство $l\left(x^{n}\right) \leqslant 2 \log n$, получаем, что

$$
l\left(y^{d^{\prime}-\hat{d}^{\prime}}, y^{b-\hat{b}}\right) \leqslant 4 \log \left(s 2^{u}\right)=4 \log s+4 u .
$$

Для оценки сложности вычисления $y^{d-d^{\prime}}$ воспользуемся легко извлекаемым из [2] (см. также [1]) неравенством

$$
l\left(x^{n}\right) \leqslant \log n+K_{1} \frac{\log n}{\log \log n},
$$

где $K_{1}$ - некоторая абсолютная константа. Учитывая, что величина $d-d^{\prime}$ может быть равна 0 , видим, что

$$
l\left(y^{d-d^{\prime}}\right) \leqslant \log \left(\max \left(d-d^{\prime}, 1\right)\right)+K_{1} \frac{\log a}{\log \log a} .
$$

Таким образом,

$$
l_{14} \leqslant \log \left(\max \left(d-d^{\prime}, 1\right)\right)+K_{1} \frac{\log a}{\log \log a}+4 \log s+4 u .
$$

V этап. Вычисление мономов

$$
x^{a} y^{b}, \quad x^{c} y^{d}
$$

На третьем этапе вычислены мономы $x^{a} y^{\hat{b}}$ и $x^{c} y^{\hat{d}^{\prime}}$, а на четвертом - мономы $y^{d^{\prime}-\hat{d}^{\prime}}$, $y^{b-\hat{b}}$ и $y^{d-d^{\prime}}$. Поэтому систему мономов $\left\{x^{a} y^{b}, x^{c} y^{d}\right\}$ можно получить, использовав 3 операции умножения, то есть

$$
l_{15} \leqslant 3 .
$$

Таким образом, в условиях рассматриваемого случая величину $l\left(x^{a} y^{b}, x^{c} y^{d}\right)$ можно сверху оценить следующим образом:

$$
\begin{aligned}
l\left(x^{a} y^{b}, x^{c} y^{d}\right) \leqslant & l_{11}+l_{12}+l_{13}+l_{14}+l_{15} \\
\leqslant & \left(2^{u}-2\right)+(s+\log a)+\left(2 s+4 \cdot 2^{u}\right) \\
& \quad+\left(\log \left(\max \left(d-d^{\prime}, 1\right)\right)+K_{1} \frac{\log a}{\log \log a}+4 \log s+4 u\right)+3 \\
& \leqslant \log a+\log \left(\max \left(d-\frac{b c}{a}, 1\right)\right)+10 \cdot 2^{u}+7 s+K_{1} \frac{\log a}{\log \log a} .
\end{aligned}
$$

Положим

$$
u=\lfloor\log \log a-2 \log \log \log a\rfloor .
$$


Учитывая неравенство $(s-1) u \leqslant \log a$, получаем, что

$$
s \leqslant \frac{\log a}{\lfloor\log \log a-2 \log \log \log a\rfloor}+1 .
$$

Поэтому окончательно в этом случае

$$
\begin{aligned}
l\left(x^{a} y^{b}, x^{c} y^{d}\right) & \leqslant \log a+\log (\max |d-b c / a|, 1)+O\left(\frac{\log a}{\log \log a}\right) \\
& \sim \log a+\log (\max |d-b c / a|, 1) \\
& \leqslant \log (|a d-b c|+a) .
\end{aligned}
$$

Во втором случае, пусть выполнено неравенство $a d<b c$.

Отметим, что в условиях этого случая выполняется неравенство $c>0$. Кроме того, тогда верно соотношение

$$
\left\lfloor\frac{d}{c}\right\rfloor \leqslant\left\lfloor\frac{b}{a}\right\rfloor .
$$

Далее положим

$$
q_{i}=\left\lfloor\frac{d}{c} 2^{i u}\right\rfloor, \quad i=0,1, \ldots, t-1
$$

Покажем, что

$$
2^{u} q_{i} \leqslant q_{i+1} \leqslant 2^{u} q_{i}+2^{u}-1, \quad i=0,1, \ldots, t-2
$$

Действительно, с одной стороны,

$$
\frac{q_{i+1}}{q_{i}}=\frac{\left\lfloor(d / c) 2^{(i+1) u}\right\rfloor}{\left\lfloor(d / c) 2^{i u}\right\rfloor} \geqslant \frac{\left.\left\lfloor(d / c) 2^{i u}\right\rfloor 2^{u}\right\rfloor}{\left\lfloor(d / c) 2^{i u}\right\rfloor}=2^{u},
$$

а с другой,

$$
q_{i+1}-2^{u} q_{i}=\left\lfloor\frac{d}{c} 2^{i u} 2^{u}\right\rfloor-\left\lfloor\frac{d}{c} 2^{i u}\right\rfloor 2^{u}<\frac{d}{c} 2^{i u} 2^{u}-\left(\frac{d}{c} 2^{i u}-1\right) 2^{u}=2^{u},
$$

и следовательно, учитывая целочисленность величины $q_{i+1}-2^{u} q_{i}$, видим, что $q_{i+1}-2^{u} q_{i} \leqslant 2^{u}-1$.

Положим

$$
\tilde{b}=\alpha_{0} q_{0}+\alpha_{1} q_{1}+\ldots+\alpha_{t-1} q_{t-1}+\alpha_{t} p_{t}+\alpha_{t+1} p_{t+1}+\ldots+\alpha_{s-1} p_{s-1} .
$$

Тогда, учитывая, что $\lfloor d / c\rfloor \leqslant\lfloor b / a\rfloor$, получаем, во-первых, что

$$
\begin{aligned}
\tilde{b} & =\alpha_{0}\left\lfloor\frac{d}{c}\right\rfloor+\alpha_{1}\left\lfloor\frac{d}{c} 2^{u}\right\rfloor+\ldots+\alpha_{t-1}\left\lfloor\frac{d}{c} 2^{(t-1) u}\right\rfloor \\
& \quad+\alpha_{t}\left\lfloor\frac{b}{a} 2^{t u}\right\rfloor+\alpha_{t+1}\left\lfloor\frac{b}{a} 2^{(t+1) u}\right\rfloor+\ldots+\alpha_{s-1}\left\lfloor\frac{b}{a} 2^{(s-1) u}\right\rfloor \\
& \leqslant \alpha_{0}\left\lfloor\frac{b}{a}\right\rfloor+\alpha_{1}\left\lfloor\frac{b}{a} 2^{u}\right\rfloor+\ldots+\alpha_{s-1}\left\lfloor\frac{b}{a} 2^{(s-1) u}\right\rfloor \\
& \leqslant \frac{b}{a}\left(\alpha_{0}+\alpha_{1} 2^{u}+\ldots+\alpha_{s-1} 2^{(s-1) u}\right)=b,
\end{aligned}
$$


а во-вторых,

$$
\begin{aligned}
\tilde{b}= & \left.\alpha_{0} \mid \frac{d}{c}\right\rfloor+\alpha_{1}\left\lfloor\frac{d}{c} 2^{u}\right\rfloor+\ldots+\alpha_{t-1}\left\lfloor\frac{d}{c} 2^{(t-1) u}\right\rfloor \\
& \left.\left.\quad+\alpha_{t}\left\lfloor\frac{b}{a} 2^{t u}\right\rfloor+\alpha_{t+1} \mid \frac{b}{a} 2^{(t+1) u}\right\rfloor+\ldots+\alpha_{s-1} \mid \frac{b}{a} 2^{(s-1) u}\right\rfloor \\
\geqslant & \alpha_{0}\left(\frac{d}{c}-1\right)+\alpha_{1}\left(\frac{d}{c} 2^{u}-1\right)+\ldots+\alpha_{t-1}\left(\frac{d}{c} 2^{(t-1) u}-1\right) \\
& \quad+\alpha_{t}\left(\frac{b}{a} 2^{t u}-1\right)+\alpha_{t+1}\left(\frac{b}{a} 2^{(t+1) u}-1\right)+\ldots+\alpha_{s-1}\left(\frac{b}{a} 2^{(s-1) u}-1\right) \\
= & \frac{d}{c}\left(\alpha_{0}+\alpha_{1} 2^{u}+\ldots+\alpha_{t-1} 2^{(t-1) u}\right) \\
& \quad+\frac{b}{a}\left(\alpha_{t} 2^{t u}+\alpha_{t+1} 2^{(t+1) u}+\ldots+\alpha_{s-1} 2^{(s-1) u}\right)-\left(\alpha_{0}+\alpha_{1}+\ldots+\alpha_{s-1}\right) \\
= & \left.\frac{d}{c}-\frac{b}{a}\right)\left(\alpha_{0}+\alpha_{1} 2^{u}+\ldots+\alpha_{t-1} 2^{(t-1) u}\right) \\
& \quad+\frac{b}{a}\left(\alpha_{0}+\alpha_{1} 2^{u}+\ldots+\alpha_{s-1} 2^{(s-1) u}\right)-\left(\alpha_{0}+\alpha_{1}+\ldots+\alpha_{s-1}\right) \\
= & b-\left(\frac{b c}{a}-d\right) \frac{\alpha_{0}+\alpha_{1} 2^{u}+\ldots+\alpha_{t-1} 2^{(t-1) u}}{c}-\left(\alpha_{0}+\alpha_{1}+\ldots+\alpha_{s-1}\right) \\
\geqslant & b-\left(\frac{b c}{a}-d\right) \frac{2^{(t-1) u} \cdot 2^{u}}{c}-s 2^{u} \geqslant b-\left(\frac{b c}{a}-d\right) 2^{u}-s 2^{u} .
\end{aligned}
$$

Оценим теперь величину $p_{t-1}-q_{t-1}$ :

$$
\begin{aligned}
p_{t-1}-q_{t-1} & =\left\lfloor\frac{b}{a} 2^{(t-1) u}\right\rfloor-\left\lfloor\frac{d}{c} 2^{(t-1) u}\right\rfloor \leqslant 2^{(t-1) u}\left(\frac{b}{a}-\frac{d}{c}\right)+1 \\
& =\left(\frac{b c}{a}-d\right) \frac{2^{(t-1) u}}{c}+1 \leqslant\left(\frac{b c}{a}-d\right)+1
\end{aligned}
$$

Эти соотношения вместе с очевидным неравенством $q_{t-1} \leqslant p_{t-1}$ дают оценки

$$
p_{t-1}-\left(\left(\frac{b c}{a}-d\right)+1\right) \leqslant q_{t-1} \leqslant p_{t-1} \text {. }
$$

Положим

$$
\tilde{d}=\gamma_{0} q_{0}+\gamma_{1} q_{1}+\ldots+\gamma_{t-1} q_{t-1}
$$

Оценим величину $\tilde{d}$. С одной стороны,

$$
\begin{aligned}
\tilde{d} & =\gamma_{0}\left\lfloor\frac{d}{c}\right\rfloor+\gamma_{1}\left\lfloor\frac{d}{c} 2^{u}\right\rfloor+\ldots+\gamma_{t-1}\left\lfloor\frac{d}{c} 2^{(t-1) u}\right\rfloor \\
& \leqslant \frac{d}{c}\left(\gamma_{0}+\gamma_{1} 2^{u}+\ldots+\gamma_{t-1} 2^{(t-1) u}\right)=d
\end{aligned}
$$


а с другой стороны,

$$
\begin{aligned}
\tilde{d} & =\gamma_{0}\left\lfloor\frac{d}{c}\right\rfloor+\gamma_{1}\left\lfloor\frac{d}{c} 2^{u}\right\rfloor+\ldots+\gamma_{t-1}\left\lfloor\frac{d}{c} 2^{(t-1) u}\right\rfloor \\
& \geqslant \gamma_{0}\left(\frac{d}{c}-1\right)+\gamma_{1}\left(\frac{d}{c} 2^{u}-1\right)+\ldots+\gamma_{t-1}\left(\frac{d}{c} 2^{(t-1) u}-1\right) \\
& =\frac{d}{c}\left(\gamma_{0}+\gamma_{1} 2^{u}+\ldots+\gamma_{t-1} 2^{(t-1) u}\right)-\left(\gamma_{0}+\gamma_{1}+\ldots+\gamma_{t-1}\right)>d-t 2^{u} .
\end{aligned}
$$

Следовательно,

$$
d-t 2^{u}<\tilde{d} \leqslant d .
$$

Перейдем непосредственно к описанию процесса совместного вычисления мономов $x^{a} y^{b}$ и $x^{c} y^{d}$ в условиях рассматриваемого случая. Это вычисление, как и в предыдущем случае, будет состоять из пяти этапов. Обозначим через $l_{2 i}, i=1,2,3,4,5$, число операций умножения, используемых на $i$-м этапе.

I этап. Вычисление системы степеней

$$
y, y^{2}, y^{3}, \ldots, y^{2^{u}-1}
$$

Очевидно, что

$$
l_{21}=2^{u}-2
$$

II этап. Вычисление степеней

$$
y^{d-\tilde{d}}, \quad y^{p_{t-1}-q_{t-1}}, \quad y^{b-\tilde{b}} .
$$

Учитывая соотношения

$$
0 \leqslant d-\tilde{d} \leqslant t 2^{u} \leqslant s 2^{u}
$$

и используя очевидное неравенство $l\left(x^{n}\right) \leqslant 2 \log n$, получаем оценку

$$
l\left(y^{d-\tilde{d}}\right) \leqslant 2 \log \left(s 2^{u}\right)=2 \log s+2 u .
$$

В силу неравенства $b-\tilde{b} \leqslant(b c / a-d) 2^{u}+s 2^{u}$, степень $y^{b-\tilde{b}}$ можно представить в виде $y^{b-\tilde{b}}=y^{r_{1}} y^{r_{2}}$, где $0 \leqslant r_{1} \leqslant(b c / a-d) 2^{u}, 0 \leqslant r_{2} \leqslant s 2^{u}, r_{1}$ и $r_{2}$ - целые числа. Очевидно, что

$$
l\left(y^{r_{2}}\right) \leqslant 2 u+2 \log s .
$$

В силу неравенства $p_{t-1}-q_{t-1} \leqslant(b c / a-d)+1$, степень $y^{p_{t-1}-q_{t-1}}$ можно представить в виде $y^{p_{t-1}-q_{t-1}}=y^{r_{3}} y^{r_{4}}$, где $0 \leqslant r_{3} \leqslant(b c / a-d)+1,0 \leqslant r_{4} \leqslant 1, r_{3}$ и $r_{4}$ - целые числа.

Теперь оценим сложность совместной реализации степеней $y^{r_{1}}$ и $y^{r_{3}}$. Отметим, что $\max \left(r_{1}, r_{3}\right) \leqslant(b c / a-d) 2^{u}$.

Применяя теорему 1 из [6], получаем, что

$$
\begin{aligned}
l\left(y^{r_{1}}, y^{r_{3}}\right) & \leqslant \log \left(\max \left(\left(\frac{b c}{a}-d\right) 2^{u}, 1\right)\right)+K_{2} \frac{\log a}{\log \log a} \\
& \leqslant u+\log \left(\max \left(\frac{b c}{a}-d, 1\right)\right)+K_{2} \frac{\log a}{\log \log a}
\end{aligned}
$$


где $K_{2}$ - некоторая абсолютная константа. Отметим, что эту же оценку можно получить из оценки сложности в случае 1 , положив $b$ и $d$ равными 1 и затем игнорировав переменную $y$.

Таким образом, на втором этапе окончательно получаем, что

$$
\begin{aligned}
l_{22} \leqslant & (2 \log s+2 u)+(2 \log s+2 u) \\
& +\left(u+\log \left(\max \left(\frac{b c}{a}-d, 1\right)\right)+K_{2} \frac{\log a}{\log \log a}\right)+2 \\
& \leqslant \log \left(\max \left(\frac{b c}{a}-d, 1\right)\right)+K_{2} \frac{\log a}{\log \log a}+7 u+4 \log s .
\end{aligned}
$$

III этап. Вычисление мономов

$$
x y^{q_{0}}, x^{2^{u}} y^{q_{1}}, \ldots, x^{2^{(t-1) u}} y^{q_{t-1}}, \quad x^{2^{(t-1) u}} y^{p_{t-1}}, x^{2^{t u}} y^{p_{t}}, \ldots, x^{2^{(s-1) u}} y^{p_{s-1}} .
$$

Сначала, учитывая, что $q_{0}$ равно либо 0 , либо 1 , последовательно вычисляем

$$
x y^{q_{0}},\left(x y^{q_{0}}\right)^{2},\left(x y^{q_{0}}\right)^{4}, \ldots,\left(x y^{q_{0}}\right)^{2^{u}} .
$$

Теперь, учитывая справедливость неравенств

$$
q_{0} 2^{u} \leqslant q_{1} \leqslant q_{0} 2^{u}+2^{u}-1 \text {, }
$$

а также тот факт, что все степени $y$, не превышающие величины $2^{u}-1$, уже вычислены на первом этапе, с использованием одной операции умножения получаем моном

$$
x^{2^{u}} y^{q_{1}}=x^{2^{u}} y^{q_{0} 2^{u}} y^{q_{1}-q_{0} 2^{u}}=\left(x y^{q_{0}}\right)^{2^{u}} y^{q_{1}-q_{0} 2^{u}} .
$$

Далее аналогично последовательно вычисляем мономы

$$
\begin{gathered}
\left(x^{2^{u}} y^{q_{1}}\right)^{2},\left(x^{2^{u}} y^{q_{1}}\right)^{4}, \ldots,\left(x^{2^{u}} y^{q_{1}}\right)^{2^{u}}, x^{2^{2 u}} y^{q_{2}}, \\
\left(x^{2^{2 u}} y^{q_{2}}\right)^{2},\left(x^{2^{2 u}} y^{q_{2}}\right)^{4}, \ldots,\left(x^{2^{2 u}} y^{q_{2}}\right)^{2^{u}}, x^{2^{3 u}} y^{q_{3}}, \\
\ldots \\
\left(x^{2^{(t-2) u}} y^{q_{t-2}}\right)^{2},\left(x^{2^{(t-2) u}} y^{q_{t-2}}\right)^{4}, \ldots,\left(x^{2^{(t-2) u}} y^{q_{t-2}}\right)^{2^{u}}, x^{2^{(t-1) u}} y^{q_{t-1}} .
\end{gathered}
$$

Умножив последний моном на вычисленную на втором этапе степень $y^{p_{t-1}-q_{t-1}}$, получаем моном $x^{2^{(t-1) u}} y^{p_{t-1}}$. Теперь последовательно вычисляем мономы

$$
\begin{gathered}
\left(x^{2^{(t-1) u}} y^{p_{t-1}}\right)^{2},\left(x^{2^{(t-1) u}} y^{p_{t-1}}\right)^{4}, \ldots,\left(x^{2^{(t-1) u}} y^{p_{t-1}}\right)^{2^{u}}, x^{2^{t u}} y^{p_{t}}, \\
\left(x^{2^{t u}} y^{p_{t}}\right)^{2},\left(x^{2^{t u}} y^{p_{t}}\right)^{4}, \ldots,\left(x^{2^{t u}} y^{p_{t}}\right)^{2^{u}}, x^{2^{(t+1) u}} y^{p_{t+1}}, \\
\ldots \\
\left(x^{2^{(s-2) u}} y^{p_{s-2}}\right)^{2},\left(x^{2^{(s-2) u}} y^{p_{s-2}}\right)^{4}, \ldots,\left(x^{2^{(s-2) u}} y^{p_{s-2}}\right)^{2^{u}}, x^{2^{(s-1) u}} y^{p_{s-1}} .
\end{gathered}
$$

Для каждого нового монома, получаемого на третьем этапе, использовалась одна операция умножения. Поэтому $l_{23} \leqslant(s-1)(u+1)$. Учитывая, что $(s-1) u \leqslant \log a$, получаем оценку

$$
l_{23} \leqslant \log a+s .
$$


IV этап. Вычисление мономов

$$
x^{a} y^{\tilde{b}}, \quad x^{c} y^{\tilde{d}}
$$

Введем множества индексов $I_{1}^{1}, I_{2}^{1}, \ldots, I_{2^{u}-1}^{1}, I_{1}^{2}, I_{2}^{2}, \ldots, I_{2^{u}-1}^{2}$ и $J_{1}, J_{2}, \ldots, J_{2^{u}-1}$ следующим образом:

$$
\begin{array}{ll}
I_{m}^{1}=\left\{i \mid(0 \leqslant i \leqslant t-1) \&\left(\alpha_{i}=m\right)\right\}, & I_{m}^{2}=\left\{i \mid(t \leqslant i \leqslant s-1) \&\left(\alpha_{i}=m\right)\right\}, \\
J_{m}=\left\{i \mid(0 \leqslant i \leqslant t-1) \&\left(\gamma_{i}=m\right)\right\}, & m=1,2, \ldots, 2^{u}-1 .
\end{array}
$$

Отметим, что хотя бы одно из множеств индексов $I_{m}^{2}, m=1,2, \ldots, 2^{u}-1$, непусто, так как $\alpha_{s-1} \neq 0$. Аналогично, непусто хотя бы одно из множеств $J_{m}, m=1,2, \ldots, 2^{u}-1$. Кроме того, отметим справедливость неравенств

$$
\sum_{i=1}^{2^{u}-1}\left|I_{i}^{1}\right|+\sum_{i=1}^{2^{u}-1}\left|I_{i}^{2}\right| \leqslant s, \quad \sum_{i=1}^{2^{u}-1}\left|J_{i}\right| \leqslant t
$$

Последовательно определим мономы

$$
f_{2^{u}-1}(x, y), g_{2^{u}-1}(x, y), f_{2^{u}-2}(x, y), g_{2^{u-2}}(x, y), \ldots, f_{1}(x, y), g_{1}(x, y) .
$$

Сначала положим

$$
\begin{aligned}
& f_{2^{u-1}}(x, y)=\prod_{i \in I_{2^{u}-1}^{1}}\left(x^{2^{i u}} y^{q_{i}}\right) \prod_{i \in I_{2^{u}-1}^{2}}\left(x^{2^{i u}} y^{p_{i}}\right), \\
& g_{2^{u}-1}(x, y)=\prod_{i \in J_{2^{u}-1}}\left(x^{2^{i u}} y^{q_{i}}\right) .
\end{aligned}
$$

Далее, для $m=2^{u}-2,2^{u}-3, \ldots, 1$, положим

$$
\begin{aligned}
& f_{m}(x, y)=f_{m+1}(x, y) \prod_{i \in I_{m}^{1}}\left(x^{2^{i u}} y^{q_{i}}\right) \prod_{i \in I_{m}^{2}}\left(x^{2^{i u}} y^{p_{i}}\right), \\
& g_{m}(x, y)=g_{m+1}(x, y) \prod_{i \in J_{m}}\left(x^{2^{i u}} y^{q_{i}}\right) .
\end{aligned}
$$

Теперь, считая, что произведение пустого множества сомножителей по определению равно единице, вычислим последовательно все отличные от единицы мономы

$$
\begin{aligned}
& \prod_{i \in I_{2^{u}-1}^{1}}\left(x^{2^{i u}} y^{q_{i}}\right) \prod_{i \in I_{2^{u}-1}^{2}}\left(x^{2^{i u}} y^{p_{i}}\right), \\
& \prod_{i \in I_{2^{u}-2}^{1}}\left(x^{2^{i u}} y^{q_{i}}\right) \prod_{i \in I_{2^{u}-2}^{2}}\left(x^{2^{i u}} y^{p_{i}}\right), \ldots, \prod_{i \in I_{1}^{1}}\left(x^{2^{i u}} y^{q_{i}}\right) \prod_{i \in I_{1}^{2}}\left(x^{2^{i u}} y^{p_{i}}\right), \\
& \prod_{i \in J_{2^{u}-1}}\left(x^{2^{i u}} y^{p_{i}}\right), \prod_{i \in J_{2^{u}-2}}\left(x^{2^{i u}} y^{p_{i}}\right), \ldots, \prod_{i \in J_{1}}\left(x^{2^{i u}} y^{p_{i}}\right) .
\end{aligned}
$$

На вычисление этих мономов потребуется не более $s+t$ операций умножения. 
Далее, используя по одной операции на каждый новый моном, вычислим

$$
\begin{aligned}
& f_{2^{u-1}}(x, y), f_{2^{u}-2}(x, y), \ldots, f_{1}(x, y), \\
& f_{2^{u}-1}(x, y) f_{2^{u}-2}(x, y), \\
& f_{2^{u}-1}(x, y) f_{2^{u}-2}(x, y) f_{2^{u}-3}(x, y), \ldots, f_{2^{u}-1}(x, y) f_{2^{u}-2}(x, y) \ldots f_{1}(x, y), \\
& g_{2^{u}-1}(x, y), g_{2^{u}-2}(x, y), \ldots, g_{1}(x, y), \\
& g_{2^{u}-1}(x, y) g_{2^{u}-2}(x, y), \\
& g_{2^{u}-1}(x, y) g_{2^{u}-2}(x, y) g_{2^{u}-3}(x, y), \ldots, g_{2^{u}-1}(x, y) g_{2^{u}-2}(x, y) \ldots g_{1}(x, y) .
\end{aligned}
$$

Тем самым вычислены мономы $x^{a} y^{\tilde{b}}$ и $x^{c} y^{\tilde{d}}$. Действительно,

$$
\begin{aligned}
& f_{2^{u}-1}(x, y) f_{2^{u}-2}(x, y) \ldots f_{1}(x, y)=\left(\prod_{i \in I_{2^{u}-1}^{1}}\left(x^{2^{i u}} y^{q_{i}}\right) \prod_{i \in I_{2^{u}-1}^{2^{u}}}\left(x^{2^{i u}} y^{p_{i}}\right)\right)^{2^{u}-1} \\
& \times\left(\prod_{i \in I_{2^{u}-2}^{1}}\left(x^{2^{i u}} y^{q_{i}}\right) \prod_{i \in I_{2^{u}-2}^{2}}\left(x^{2^{i u}} y^{p_{i}}\right)\right)^{2^{u}-2} \cdots\left(\prod_{i \in I_{1}^{1}}\left(x^{2^{i u}} y^{q_{i}}\right) \prod_{i \in I_{1}^{2}}\left(x^{2^{i u}} y^{p_{i}}\right)\right)^{1} \\
& =\left(\prod_{i \in I_{2^{u}-1}^{1}}\left(x^{2^{i u}} y^{q_{i}}\right)^{\alpha_{i}} \prod_{i \in I_{2^{u}-1}^{2}}\left(x^{2^{i u}} y^{p_{i}}\right)^{\alpha_{i}}\right) \\
& \times\left(\prod_{i \in I_{2^{u}-2}^{1}}\left(x^{2^{i u}} y^{q_{i}}\right)^{\alpha_{i}} \prod_{i \in I_{2^{u}-2}^{2}}\left(x^{2^{i u}} y^{p_{i}}\right)^{\alpha_{i}}\right) \ldots\left(\prod_{i \in I_{1}^{1}}\left(x^{2^{i u}} y^{q_{i}}\right)^{\alpha_{i}} \prod_{i \in I_{1}^{2}}\left(x^{2^{i u}} y^{p_{i}}\right)^{\alpha_{i}}\right) \\
& =\left(\prod_{i: \alpha_{i} \neq 0} x^{\alpha_{i} 2^{i u}}\right)\left(\prod_{i=0}^{t-1} y^{\alpha_{i} q_{i}}\right)\left(\prod_{i=t}^{s-1} y^{\alpha_{i} p_{i}}\right) \\
& =x^{\alpha_{0}+\alpha_{1} 2^{u}+\ldots+\alpha_{s-1} 2^{(s-1) u}} y^{\alpha_{0} q_{0}+\alpha_{1} q_{1}+\ldots+\alpha_{t-1} q_{t-1}+\alpha_{t} p_{t}+\alpha_{t+1} p_{t+1}+\ldots+\alpha_{t-1} p_{t-1}}=x^{a} y^{\tilde{b}} \text {, } \\
& g_{2^{u-1}}(x, y) g_{2^{u-2}}(x, y) \ldots g_{1}(x, y) \\
& =\left(\prod_{i \in J_{2} u-1}\left(x^{2^{i u}} y^{q_{i}}\right)\right)^{2^{u}-1}\left(\prod_{i \in J_{2} u-2}\left(x^{2^{i u}} y^{q_{i}}\right)\right)^{2^{u}-2} \ldots\left(\prod_{i \in J_{1}}\left(x^{2^{i u}} y^{q_{i}}\right)\right)^{1} \\
& =\left(\prod_{i \in J_{2} u_{-1}}\left(x^{2^{i u}} y^{q_{i}}\right)^{\gamma_{i}}\right)\left(\prod_{i \in J_{2^{u}-2}}\left(x^{2^{i u}} y^{q_{i}}\right)^{\gamma_{i}}\right) \ldots\left(\prod_{i \in J_{1}}\left(x^{2^{i u}} y^{q_{i}}\right)^{\gamma_{i}}\right) \\
& =\prod_{i: \gamma_{i} \neq 0}\left(x^{\gamma_{i} 2^{i u}} y^{\gamma_{i} q_{i}}\right)=x^{\gamma_{0}+\gamma_{1} 2^{u}+\ldots+\gamma_{t-1} 2^{(t-1) u}} y^{\gamma_{0} q_{0}+\gamma_{1} q_{1}+\ldots+\gamma_{t-1} q_{t-1}}=x^{c} y^{\tilde{d}} \text {. }
\end{aligned}
$$

Очевидно, что

$$
l_{24} \leqslant\left(s+2 \cdot 2^{u}\right)+\left(t+2 \cdot 2^{u}\right) \leqslant 2 s+4 \cdot 2^{u} .
$$

V этап. Вычисление мономов

$$
x^{a} y^{b}, \quad x^{c} y^{d}
$$


На втором этапе вычислены степени $y^{b-\tilde{b}}$ и $y^{d-\tilde{d}}$, а на четвертом - мономы $x^{a} y^{\tilde{b}}$ и $x^{c} y^{\tilde{d}}$. Поэтому систему мономов $\left\{x^{a} y^{b}, x^{c} y^{d}\right\}$ можно получить, использовав 2 операции умножения, то есть

$$
l_{25} \leqslant 2 \text {. }
$$

Таким образом, во втором случае величину $l\left(x^{a} y^{b}, x^{c} y^{d}\right)$ можно сверху оценить следующим образом:

$$
\begin{aligned}
l\left(x^{a} y^{b}, x^{c} y^{d}\right) \leqslant & l_{21}+l_{22}+l_{23}+l_{24}+l_{25} \\
\leqslant & \left(2^{u}-2\right)+\left(\log \left(\max \left(\frac{b c}{a}-d, 1\right)\right)+K_{2} \frac{\log a}{\log \log a}+4 \log s+7 u\right) \\
& \quad+(s+\log a)+\left(2 s+4 \cdot 2^{u}\right)+2 \\
& \leqslant \log a+\log \left(\max \left(\frac{b c}{a}-d, 1\right)\right)+12 \cdot 2^{u}+7 s+K_{2} \frac{\log a}{\log \log a} .
\end{aligned}
$$

Положим

$$
u=\lfloor\log \log a-2 \log \log \log a\rfloor .
$$

Тогда, учитывая, что $(s-1) u \leqslant \log a$, получаем неравенство

$$
s \leqslant \frac{\log a}{\lfloor\log \log a-2 \log \log \log a\rfloor}+1 .
$$

Поэтому во втором случае окончательно получаем, что

$$
\begin{aligned}
l\left(x^{a} y^{b}, x^{c} y^{d}\right) & \leqslant \log a+\log \left(\max \left|\frac{b c}{a}-d\right|, 1\right)+O\left(\frac{\log a}{\log \log a}\right) \\
& \sim \log a+\log \left(\max \left|\frac{b c}{a}-d\right|, 1\right) \leqslant \log (|a d-b c|+a) .
\end{aligned}
$$

Верхняя оценка доказана.

Из теоремы 1 можно извлечь тот факт, что асимптотически почти все системы мономов $\left\{x^{a} y^{b}, x^{c} y^{d}\right\}$ имеют максимальную сложность $(2+o(1)) \log \max \{a, b, c, d\}$. Верхняя оценка непосредственно следует из теоремы 1. Приведем утверждение о нижней оценке.

Следствие 1. Среди всех систем мономов $\left\{x^{a} y^{b}, x^{c} y^{d}\right\}, y$ которых показатели степеней $a, b, c$ и $d$ не превосходят $n$, доля систем, удовлетворяющих неравенству

$$
l\left(x^{a} y^{b}, x^{c} y^{d}\right) \geqslant 2 \log n-\log \log n,
$$

стреитеся к 1 при $n \rightarrow \infty$.

Доказательство. С одной стороны, среди всех систем мономов $\left\{x^{a} y^{b}, x^{c} y^{d}\right\}$, где $a, b, c$, $d$ - целые числа, не превосходящие $n$, доля систем, у которых все показатели степени $a$, $b, c, d$ не менее величины $n /(\log n)^{1 / 3}$, стремится к 1 при $n \rightarrow \infty$.

С другой стороны, при фиксированных значениях $a, b$ и $c$, удовлетворяющих неравенствам

$$
\frac{n}{(\log n)^{1 / 3}} \leqslant a \leqslant n, \quad \frac{n}{(\log n)^{1 / 3}} \leqslant b \leqslant n, \quad \frac{n}{(\log n)^{1 / 3}} \leqslant c \leqslant n
$$


положив $d_{0}=(b c) / a$, получаем, что доля целых $d$ из отрезка $\left[n /(\log n)^{1 / 3}, n\right]$, удовлетворяющих неравенству

$$
\left|d-d_{0}\right| \geqslant \frac{n}{(\log n)^{1 / 3}},
$$

стремится к 1 при $n \rightarrow \infty$. Тогда, в случае выполнения этого неравенства, в силу леммы 5 справедлива нижняя оценка

$$
\begin{aligned}
l\left(x^{a} y^{b}, x^{c} y^{d}\right) & \geqslant \log (|a d-b c|+a)-1 \geqslant \log \left|a\left(d-d_{0}+d_{0}\right)-b c\right|-1 \\
& =\log \left(a\left|d-d_{0}\right|\right)-1 \geqslant \log \left(\frac{n}{(\log n)^{1 / 3}} \frac{n}{(\log n)^{1 / 3}}\right)-1 \geqslant 2 \log n-\log \log n .
\end{aligned}
$$

Следствие доказано.

\section{Список литературы}

1. Кнут Д. Е., Искусство програмиирования для ЭВМ, т. 2. Мир, Москва, 1977.

2. Brauer A., On addition chains. Bull. Amer. Math. Soc. (1939) 45, 736-739.

3. Bellman R. E., Addition chains of vectors. Amer. Math. Monthly (1963) 70, 765.

4. Straus E. G., Addition chains of vectors. Amer. Math. Monthly (1964) 71, 806-808.

5. Yao A. C.-C., On the evaluation of powers. SIAM J. Comput. (1976) 5, 100-103.

6. Гашков С. Б., Кочергин В. В., Об аддитивных цепочках векторов, вентильных схемах и сложности вычисления степеней. Методы дискретного анализа в теории графов и сложности (1992) 52, 22-40.

7. Кочергин В. В., О сложности вычислений одночленов и наборов степеней. Дискретный анализ (1994) 27, 94-107.

8. Кочергин В. В., О двух обобщениях задачи об аддитивных цепочках. В сб.: Tруды IV Mежсдународной конференции «Дискретные модели в теории управляющих систем». МАКС, Москва, 2000, c. 55-59.

9. Pippenger N., On evaluation of powers and monomials. SIAM J. Comput. (1980) 9, №2, 230-250.

10. Кочергин В. В., О сложности вычисления систем одночленов с ограничениями на степени переменных. Дискретная математика (1998) 10, №3, 27-34.

11. Кочергин В. В., О порядке роста сложности вычисления систем одночленов от многих переменных. В сб.: Материалы Сибирской конференчии по исследованию операчий SCOR'98. Новосибирск, 1998, с. 129.

12. Кочергин В. В., О сложности вычисления системы одночленов специального вида. В сб.: Материалы Х Межсгосударственной иколы-семинара "Синтез и сложность управлющих систем». Изд-во центра прикладных исследований при механико-математическом факультете МГУ, Москва, 2000, с. 12-14.

13. Кочергин В. В., О некоторых обобщениях задачи об аддитивных цепочках. В кн.: Дискретная математика и ее приложсения. Сборник лекчий. Изд-во Центра прикладных исследований при механико-математическом факультете МГУ, Москва, 2001, с. 59-83.

14. Севидж Д. Е., Сложность вычислений. Факториал, Москва, 1998.

15. Лупанов О. Б., Об одном подходе к синтезу управляющих систем - принципе локального кодирования. Проблемы кибернетики (1965) 14, 31-110. 